DCPT $/ 10 / 162$

DESY $10-217$

$\operatorname{IPPP} / 10 / 81$

arXiv:1012.5007 [hep-ph]

\title{
BSM Higgs Physics in the Exclusive Forward Proton Mode at the LHC
}

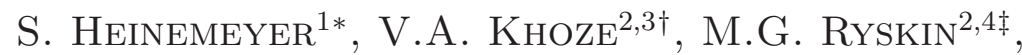

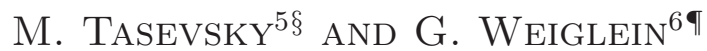 \\ ${ }^{1}$ Instituto de Física de Cantabria (CSIC-UC), Santander, Spain \\ ${ }^{2}$ IPPP, Department of Physics, Durham University, Durham DH1 3LE, U.K. \\ ${ }^{3}$ School of Physics \& Astronomy, University of Manchester, Manchester M13 9PL, U.K. \\ ${ }^{4}$ Petersburg Nuclear Physics Institute, Gatchina, St. Petersburg, 188300, Russia \\ ${ }^{5}$ Institute of Physics, 18221 Prague 8, Czech Republic \\ ${ }^{6}$ DESY, Notkestraße 85, D-22603 Hamburg, Germany
}

\begin{abstract}
We investigate the prospects for Central Exclusive Diffractive (CED) production of BSM Higgs bosons at the LHC using forward proton detectors installed at $220 \mathrm{~m}$ and $420 \mathrm{~m}$ distance around ATLAS and / or CMS. We update a previous analysis for the MSSM taking into account improvements in the theoretical calculations and the most recent exclusion bounds from the Tevatron. We extend the MSSM analysis to new benchmark scenarios that are in agreement with the cold dark matter relic abundance and other precision measurements. We analyse the exclusive production of Higgs bosons in a model with a fourth generation of fermions. Finally, we comment on the determination of Higgs spin-parity and coupling structures at the LHC and show that the forward proton mode could provide crucial information on the $\mathcal{C P}$ properties of the Higgs bosons.
\end{abstract}

*email: Sven.Heinemeyer@cern.ch

†email: V.A.Khoze@durham.ac.uk

$\ddagger$ email: misha.ryskin@durham.ac.uk

§email: Marek.Tasevsky@cern.ch

ף email: Georg.Weiglein@desy.de 


\section{Introduction}

There has been a growing interest in the recent years in the possibility to complement the standard LHC physics menu by forward and diffraction physics. This requires the installation of near-beam proton detectors in the LHC tunnel, see for example Refs. [1 5]. Within ATLAS and CMS projects to install the proton detectors at $220 \mathrm{~m}$ and $420 \mathrm{~m}$ from the interaction points have been discussed [6 8]. The combined detection of the centrally produced system and both outgoing protons gives access to a rich programme of studies of QCD, electroweak and BSM physics, see for instance Refs. [2,7,9 12. Importantly, these measurements will provide valuable information on the Higgs sector of MSSM and other popular BSM scenarios [13 19].

As it is well known, many models of new physics require an extended Higgs sector. The most popular extension of the SM is the MSSM [20, where the Higgs sector consists of five physical states. At lowest order the MSSM Higgs sector is $\mathcal{C} \mathcal{P}$-conserving, containing two $\mathcal{C P}$-even bosons, the lighter $h$ and the heavier $H$, a $\mathcal{C P}$-odd boson, $A$, and the charged bosons $H^{ \pm}$. It can be specified in terms of the gauge couplings, the ratio of the two vacuum expectation values, $\tan \beta \equiv v_{2} / v_{1}$, and the mass of the $A$ boson, $M_{A}$. The Higgs sector of the MSSM is affected by large higher-order corrections (see for example Ref. [21] for recent reviews), which have to be taken into account for reliable phenomenological predictions.

Another very simple example of physics beyond the SM is a model which extends the SM by a fourth generation of heavy fermions (SM4), see for instance Refs. [22 26]. Here the masses of the 4th generation quarks are assumed to be (much) heavier than the mass of the top-quark. In this case, the effective coupling of the Higgs boson to two gluons is three times larger than in the SM, and all branching ratios change correspondingly.

Proving that a detected new state is, indeed, a Higgs boson and distinguishing the Higgs boson(s) of the SM, the SM4 or the MSSM from the states of other theories will be far from trivial. In particular, it will be of utmost importance to determine the spin and $\mathcal{C P}$ properties of a new state and to measure precisely its mass, width and couplings.

Forward proton detectors installed at $220 \mathrm{~m}$ and $420 \mathrm{~m}$ around ATLAS and / or CMS would provide a rich complementary physics potential to the "conventional" LHC Higgs production channels. The CED processes are of the form

$$
p p \rightarrow p \oplus H \oplus p
$$

where the $\oplus$ signs denote large rapidity gaps on either side of the centrally produced state. If the outgoing protons remain intact and scatter through small angles then, to a very good approximation, the primary di-gluon system obeys a $J_{z}=0, \mathcal{C}$-even, $\mathcal{P}$-even selection rule [27]. Here $J_{z}$ is the projection of the total angular momentum along the proton beam axis. This permits a clean determination of the quantum numbers of the observed resonance which will be dominantly produced in a $0^{++}$state. Furthermore, due to the exclusive nature of the process, the proton energy losses are directly related to the central mass. This property together with precise tracking detectors provides a potentially excellent mass resolution irrespective of the decay channel (and, depending on the Higgs mass, could also provide access to a determination of the Higgs boson width). The CED processes allow in principle all the main Higgs decay modes, $b \bar{b}, W W$ and $\tau \tau$, to be observed in the same production channel. In particular, a unique possibility opens up to study the Higgs Yukawa coupling to bottom 
quarks, which, as it is well known, may be difficult to access in other search channels at the LHC. Here it should be kept in mind that access to the bottom Yukawa coupling will be crucial as an input also for the determination of Higgs couplings to other particles [28, 29].

Within the MSSM, CED Higgs production is even more appealing than in the SM. The lightest MSSM Higgs-boson coupling to $b \bar{b}$ and $\tau \tau$ can be strongly enhanced for large values of $\tan \beta$ and relatively small $M_{A}$. On the other hand, for larger values of $M_{A}$ the branching ratio of $H \rightarrow b \bar{b}$ is much larger than for a SM Higgs of the same mass. As a consequence, CED $H \rightarrow b \bar{b}$ production can be studied in the MSSM up to much higher masses than in the SM case.

The outline of the paper is as follows. We revisit the analysis of Ref. [14] where a detailed study of the CED MSSM Higgs production was performed (see also Refs. [13, 15, 30, 33] for other CED studies in the MSSM). This is updated by taking into account recent theoretical developments in background evaluation [34,35] and using an improved version of the code FeynHiggs 36 39] (available from Ref. [40]) employed for the cross section and decay width calculations. The regions excluded by LEP and Tevatron Higgs searches are evaluated with HiggsBounds [41]. These improvements are applied for the CED production of MSSM Higgs bosons [14] in the $M_{h}^{\max }$ and no-mixing benchmark scenarios (defined in [42]) as well as for new benchmark scenarios that yield the correct amount of the cold dark matter abundance. In Sect. 3 we show the corresponding results for CED Higgs production in the SM4. The determination of spin-parity quantum numbers of Higgs bosons at the LHC and the advantages provided by the CED process is briefly discussed in Sect. 4. Finally, Sect. 5 contains our conclusions.

\section{Luminosity scenarios}

Analogous to Refs. [14, 17, 19], in our numerical analysis below we consider four scenarios for the integrated luminosities and the experimental conditions for CED processes at the LHC. In order to illustrate the physics gain that could be expected if a more optimistic scenario were to be realized (see the discussion in Sect. 5 of Ref. [14]), we also include in the following a discussion of the cross sections and experimental efficiencies with scenarios where the event rates are higher by a factor of 2 (due to improvements on the experimental side and possibly higher signal rates), denoted by "eff $\times 2$ ". We furthermore assume for sake of simplicity a center-of-mass energy of $\sqrt{s}=14 \mathrm{TeV}$ at the LHC. Lower energies and correspondingly lower cross sections would require correspondingly higher luminosities.

- $\underline{60 \mathrm{fb}^{-1}}$ :

An integrated LHC luminosity of $\mathcal{L}=2 \times 30 \mathrm{fb}^{-1}$, corresponding roughly to three years of running at an instantaneous luminosity $\mathcal{L} \sim 10^{33} \mathrm{~cm}^{-2} \mathrm{~s}^{-1}$ by both ATLAS and CMS. With such a luminosity the effect of pile-up is not negligible but can be safely kept under control.

- $60 \mathrm{fb}^{-1} \mathrm{eff} \times 2$ :

The same integrated LHC luminosity as in the above scenario but with event rates that are higher by a factor of 2 (see the discussion of possible improvements and theoretical uncertainties in Ref. [14]). 
- $\underline{600 \mathrm{fb}^{-1}}$ :

An integrated LHC luminosity of $\mathcal{L}=2 \times 300 \mathrm{fb}^{-1}$ and the same efficiency factors as in the scenario with $\mathcal{L}=60 \mathrm{fb}^{-1}$. This corresponds roughly to three years of running at an instantaneous luminosity $\mathcal{L} \approx 10^{34} \mathrm{~cm}^{-2} \mathrm{~s}^{-1}$ by both ATLAS and CMS.

- $600 \mathrm{fb}^{-1}$ eff $\times 2$ :

The same integrated LHC luminosity as in the scenario with $\mathcal{L}=2 \times 300 \mathrm{fb}^{-1}$ but with event rates that are higher by a factor of 2 .

\section{Update of the MSSM analysis}

\subsection{Tree-level structure and higher-order corrections}

As it is well known, unlike in the SM, in the MSSM two Higgs doublets are required. One obtains five physical states, the neutral $\mathcal{C} \mathcal{P}$-even Higgs bosons $h, H$, the $\mathcal{C} \mathcal{P}$-odd Higgs boson $A$, and the charged Higgs bosons $H^{ \pm}$. Furthermore there are three unphysical Goldstone boson states, $G^{0}, G^{ \pm}$. At the tree level, the Higgs sector can be described with the help of two independent parameters, usually chosen as the ratio of the two vacuum expectation values, $\tan \beta \equiv v_{2} / v_{1}$, and $M_{A}$, the mass of the $\mathcal{C} \mathcal{P}$-odd $A$ boson.

In the decoupling limit, which is typically reached for $M_{A} \gtrsim 150 \mathrm{GeV}$ (depending on $\tan \beta$ ), the heavy MSSM Higgs bosons are nearly degenerate in mass, $M_{A} \approx M_{H} \approx M_{H^{ \pm}}$. The couplings of the neutral Higgs bosons to SM gauge bosons are proportional td 1

$$
V V h \sim \sin (\beta-\alpha), V V H \sim \cos (\beta-\alpha), \quad\left(V=Z, W^{ \pm}\right)
$$

while the coupling $V V A \equiv 0$ at tree level. In the decoupling limit one finds $\beta-\alpha \rightarrow \pi / 2$, i.e. $\sin (\beta-\alpha) \rightarrow 1, \cos (\beta-\alpha) \rightarrow 0$. Consequently, for $M_{A} \gtrsim 150 \mathrm{GeV}$ one finds the following decay patterns for the neutral MSSM Higgs bosons at tree-level:

$h$ : the light $\mathcal{C P}$-even Higgs boson has SM-like decays. Due to its upper mass limit of $M_{h} \lesssim 135 \mathrm{GeV}$ [38] (see below), a non-negligible decay to weak gauge bosons only occurs in a limited window of $M_{h}$ values close to this upper limit.

$H, A$ : compared to a SM Higgs boson with mass $M_{H^{\mathrm{SM}}} \gtrsim 150 \mathrm{GeV}$, which would decay predominantly into SM gauge bosons, the decays of $H, A$ to SM gauge bosons are strongly suppressed. In turn, the branching ratios to $b \bar{b}$ and $\tau^{+} \tau^{-}$are much larger in this mass range compared to the $\mathrm{SM}$ case. As a rule of thumb, $\mathrm{BR}(H, A \rightarrow b \bar{b}) \approx 90 \%$ and $\operatorname{BR}\left(H, A \rightarrow \tau^{+} \tau^{-}\right) \approx 10 \%$, if SUSY particles (such as charginos and neutralinos) are too heavy to be produced in the decays of $H$ and $A$.

Higher-order corrections in the MSSM Higgs sector are in general quite large. In particular, higher-order corrections give rise to an upward shift of the upper bound on the light $\mathcal{C P}$-even Higgs-boson mass from the tree-level value, $M_{h} \leq M_{Z}$, to about $M_{h} \lesssim 135 \mathrm{GeV}$ [38]. Besides the impact on the masses, large higher-order corrections also affect the Higgs-boson

\footnotetext{
${ }^{1} \alpha$ diagonalizes the $\mathcal{C} \mathcal{P}$-even Higgs sector at tree-level.
} 
couplings. For the evaluation of the theoretical predictions for the relevant observables in the MSSM Higgs sector we use the code FeynHiggs 36 39.

In order to fix our notation, we list the conventions for the inputs from the scalar top and scalar bottom sector of the MSSM: the mass matrices in the basis of the current eigenstates $\tilde{t}_{L}, \tilde{t}_{R}$ and $\tilde{b}_{L}, \tilde{b}_{R}$ are given by

$$
\begin{aligned}
\mathcal{M}_{\tilde{t}}^{2} & =\left(\begin{array}{cc}
M_{\tilde{Q}}^{2}+m_{t}^{2}+\cos 2 \beta\left(\frac{1}{2}-\frac{2}{3} s_{\mathrm{w}}^{2}\right) M_{Z}^{2} & m_{t} X_{t} \\
m_{t} X_{t} & M_{\tilde{t}_{R}}^{2}+m_{t}^{2}+\frac{2}{3} \cos 2 \beta s_{\mathrm{w}}^{2} M_{Z}^{2}
\end{array}\right), \\
\mathcal{M}_{\tilde{b}}^{2} & =\left(\begin{array}{cc}
M_{\tilde{Q}}^{2}+m_{b}^{2}+\cos 2 \beta\left(-\frac{1}{2}+\frac{1}{3} s_{\mathrm{w}}^{2}\right) M_{Z}^{2} & m_{b} X_{b} \\
m_{b} X_{b} & M_{\tilde{b}_{R}}^{2}+m_{b}^{2}-\frac{1}{3} \cos 2 \beta s_{\mathrm{w}}^{2} M_{Z}^{2}
\end{array}\right),
\end{aligned}
$$

where

$$
m_{t} X_{t}=m_{t}\left(A_{t}-\mu \cot \beta\right), \quad m_{b} X_{b}=m_{b}\left(A_{b}-\mu \tan \beta\right) .
$$

Here $A_{t}$ denotes the trilinear Higgs-stop coupling, $A_{b}$ denotes the Higgs-sbottom coupling, and $\mu$ is the higgsino mass parameter. As an abbreviation we will use

$$
M_{\mathrm{SUSY}} \equiv M_{\tilde{Q}}=M_{\tilde{t}_{R}}=M_{\tilde{b}_{R}} .
$$

The relation between the bottom-quark mass and the Yukawa coupling $h_{b}$, which also controls the interaction between the Higgs fields and the sbottom quarks, reads at lowest order $m_{b}=h_{b} v_{1}$. This relation is affected at one-loop order by large radiative corrections [43 46], leading to a replacement of

$$
m_{b} \rightarrow \frac{m_{b}}{1+\Delta_{b}}
$$

as the dominant effect (see also Refs. [47 51]). The quantity $\Delta_{b}$ exhibits a parametric dependence $\Delta_{b} \propto \tan \beta \mu$, where the explicit form of $\Delta_{b}$ in the limit of $M_{\text {SUSY }} \gg m_{t}$ and $\tan \beta \gg 1$ can be found in Ref. [43. The CED channel, $p p \rightarrow p \oplus \phi \oplus p$ with $\phi \rightarrow b \bar{b}(\phi=h, H)$ receives important contributions from the $\Delta_{b}$ corrections via the bottom Yukawa coupling. Because of the pronounced $\mu$ dependence we will show the results for different values of $\mu$.

\subsection{Updates with respect to the previous analyses}

In Ref. [14] we have presented detailed results on signal and background predictions of CED production of the $h$ and $H$. The results shown here differ from the previous ones in various respects, which we briefly summarize in the following.

\section{Calculation of signal and $b \bar{b}$ background}

Following the studies in Ref. [34] we use here a modified formula (with respect to Ref. [14]) for the overall physics background to the $0^{++}$Higgs signal in the $b \bar{b}$ mode

$$
\frac{\mathrm{d} \sigma^{B}}{\mathrm{~d} M} \approx 0.5 \mathrm{fb} / \mathrm{GeV}\left[A\left(\frac{120 \mathrm{GeV}}{M}\right)^{6}+\frac{C}{2}\left(\frac{120 \mathrm{GeV}}{M}\right)^{8}\right]
$$


with $A=0.92$ and $C=C_{\mathrm{NLO}}=0.48-0.12 \times(\ln (M / 120 \mathrm{GeV}))$. The expression (8) holds for a mass window $\Delta M=4-5 \mathrm{GeV}$ and summarizes several types of backgrounds: the prolific $g g^{P P} \rightarrow g g$ subprocess can mimic $b \bar{b}$ production due to the misidentification of the gluons as $b$ jets; an admixture of $\left|J_{z}\right|=2$ production; the radiative $g g^{P P} \rightarrow b \bar{b} g$ background; due to the non-zero $b$-quark mass there is also a contribution to the $J_{z}=0$ cross section of order $m_{b}^{2} / E_{T}^{2}$. The first term in the square brackets corresponds to the first three background sources [14], evaluated for $P_{g / b}=1.3 \%$, where $P_{g / b}$ is the probability to misidentify a gluon as a $b$-jet for a $b$-tagging efficiency of $60 \% 2$ The second term describes the background associated with bottom-mass terms in the Born amplitude. The NLO correction suppresses this contribution by a factor of about 2, or more for larger masses [34]. As discussed in detail in Ref. [14], eq. (8) is only an approximative formula. A more realistic approach is to implement all the background processes in a Monte Carlo program and to perform an analysis at the detector level (as it was done for the signal process). However none of the background processes mentioned in Ref. [14] has been implemented in any Monte Carlo event generator so far. Such a dedicated background analysis goes beyond the scope of our paper.

It should be kept in mind that the main experimental challenge of running at high luminosity, $\mathcal{L} \sim 10^{34} \mathrm{~cm}^{-2} \mathrm{~s}^{-1}$, is the effect of pile-up, which can generate fake signal events within the acceptances of the proton detectors as a result of the coincidence of two or more separate interactions in the same bunch crossing, see Refs. [6, 7, 14, 15] for details. As established in Refs. [15, 58, 59] we can expect that this overlap background can be brought under control by using dedicated fast-timing proton detectors with a few pico-second resolution (see Ref. [7]) and additional experimental cuts. Note also that in the analysis strategy of Ref. [14] the event selections and cuts were imposed such as to maximally reduce the pile-up background. Similarly to Ref. [14], based on the anticipated improvements for a reduction of the overlap backgrounds down to a tolerable level, here the pile-up effects are assumed to be negligible after applying all the cuts suppressing the pile-up. Consequently, the remaining pile-up backgrounds are not included in our numerical studies performed in the present paper.

From a perspective of an even further future, there is an idea to double the Level-1 trigger latency in CMS which would allow the detectors at $420 \mathrm{~m}$ to be included in the Level-1 trigger decision. Although no dedicated study of the efficiency of such a trigger or the effect of pileup on it has so far been made, we believe that a fair increase of the statistical significance could be expected in this case.

The signal cross section is calculated on the basis of the prediction for the production of a SM Higgs boson in CED together with an appropriate rescaling using the partial widths of the neutral $\mathcal{C} \mathcal{P}$-even Higgs bosons of the MSSM into gluons, $\Gamma(\phi \rightarrow g g)(\phi=h, H)$, as implemented in FeynHiggs (details are given in Ref. [14]). The predictions within FeynHiggs have been updated (from the version 2.3.0-beta used in Ref. [14] to the version 2.7.1 used here) by (see the "change log" in Ref. [40] for details)

- a change in the bottom Yukawa coupling employed for the bottom loop contribution. The running bottom mass is now evaluated at the scale of the bottom mass, $m_{b}\left(m_{b}\right)$. This scale choice was found to yield smaller higher-order corrections compared to the previous parametrisation in terms of $m_{b}\left(m_{t}\right)$. This change leads to a sizable enhanced $g g \rightarrow h(H)$ production rate at lower (all) $M_{A}$,

\footnotetext{
${ }^{2}$ Further improvements in the experimental analysis could allow to reduce $P_{g / b}$.
} 
- the inclusion of an improved version of the $\Delta_{b}$ corrections [51 to the bottom loop in the $\phi \rightarrow g g$ calculation: more higher-order contributions to $\Delta_{b}$ are taken into account, leading in our case to a further effective enhancement of the bottom Yukawa coupling, and thus to an enhanced $g g \rightarrow h(H)$ production rate at lower (all) $M_{A}$,

- the change to a running top mass, effectively decreasing the top-loop contribution, or, conversely, increasing the relative bottom-loop contribution, and effectively to a slightly enhanced $g g \rightarrow h(H)$ production rate at lower (all) $M_{A}$,

- further amendments having a smaller numerical impact.

The predictions have been improved in particular in the region of low $M_{A}$ and/or large $\mu$ and large $\tan \beta$.

Since the publication of Ref. [14] there were some further developments in the calculations of the CED cross-sections concerning both, the hard matrix element (see Refs. [3, 52, 53]) and the so-called soft absorptive corrections and soft-hard factorization breaking effects (see Refs. [54 56] for details and references). However, at the present stage we do not see sufficient reason to revise the result used in Ref. 14 for the calculation of the effective exclusive $g g$ luminosity, which determines the rates of signal and background events, see also Ref. [54. It is worth emphasizing in this context that the effective luminosity cancels in the signal-tobackground ratio for the non-pile-up background. Thus, although the overall uncertainty in the calculation of the CED cross section could be as large as a factor of $\sim 2.5$, the effect of this uncertainty on the Higgs discovery contours is comparatively weak. It should furthermore be noted that there is a good chance that the accuracy of the predictions will improve after the early runs at the LHC which will allow a detailed test of the theoretical formalism, see Ref. [57.

To summarize the effect of the various changes in the evaluation of the MSSM contributions to the scaling factors that we apply to the production rate of a SM Higgs boson in CED background calculations we show in the upper (lower) plot of Fig. 1 the contours for the ratio of signal events in the MSSM to those in the SM in the $h(H) \rightarrow b \bar{b}$ channel in CED production in the $M_{A}$-tan $\beta$ plane. The parameters are fixed according to the $M_{h}^{\max }$ benchmark scenario [42 with $\mu=+200 \mathrm{GeV}$ (see eq. (9) below). These plots are updated from Fig. 2 (upper plot) and Fig. 7 (upper plot) of Ref. [14].

Comparing our updated results with the plots in Ref. [14] one can see that the effect of the improved $g g \rightarrow h / H$ calculation gives rise to an increase of the MSSM CED Higgs production channel everywhere, while the size of the enhancement factor depends on the parameter region and the channel. In the case of the $h$ production and decay into $b \bar{b}$, we find an enhancement compared to our previous analysis of up to a factor of 2 for $M_{A}<140 \mathrm{GeV}$, while for $M_{A}>140 \mathrm{GeV}$ the change does not exceed the level of $10 \%$. For $H$ production and decay into $b \bar{b}$, we find an increase up to the level of $50 \%$ for $M_{A}<130 \mathrm{GeV}$, while an enhancement of up to a factor 3 is possible for larger $M_{A}$. As we will discuss below, the enhanced production rates obtained from the improved theoretical predictions for $g g \rightarrow h / H$ give rise to an enlargement of the parameter regions that can be covered with CED Higgs production processes within the MSSM at the $5 \sigma$ and the $3 \sigma$ level. On the other hand, the parameter regions excluded by the Higgs searches at the Tevatron have also increased as compared to our previous analysis. We show the contours of $5 \sigma$ and $3 \sigma$ significances for the 

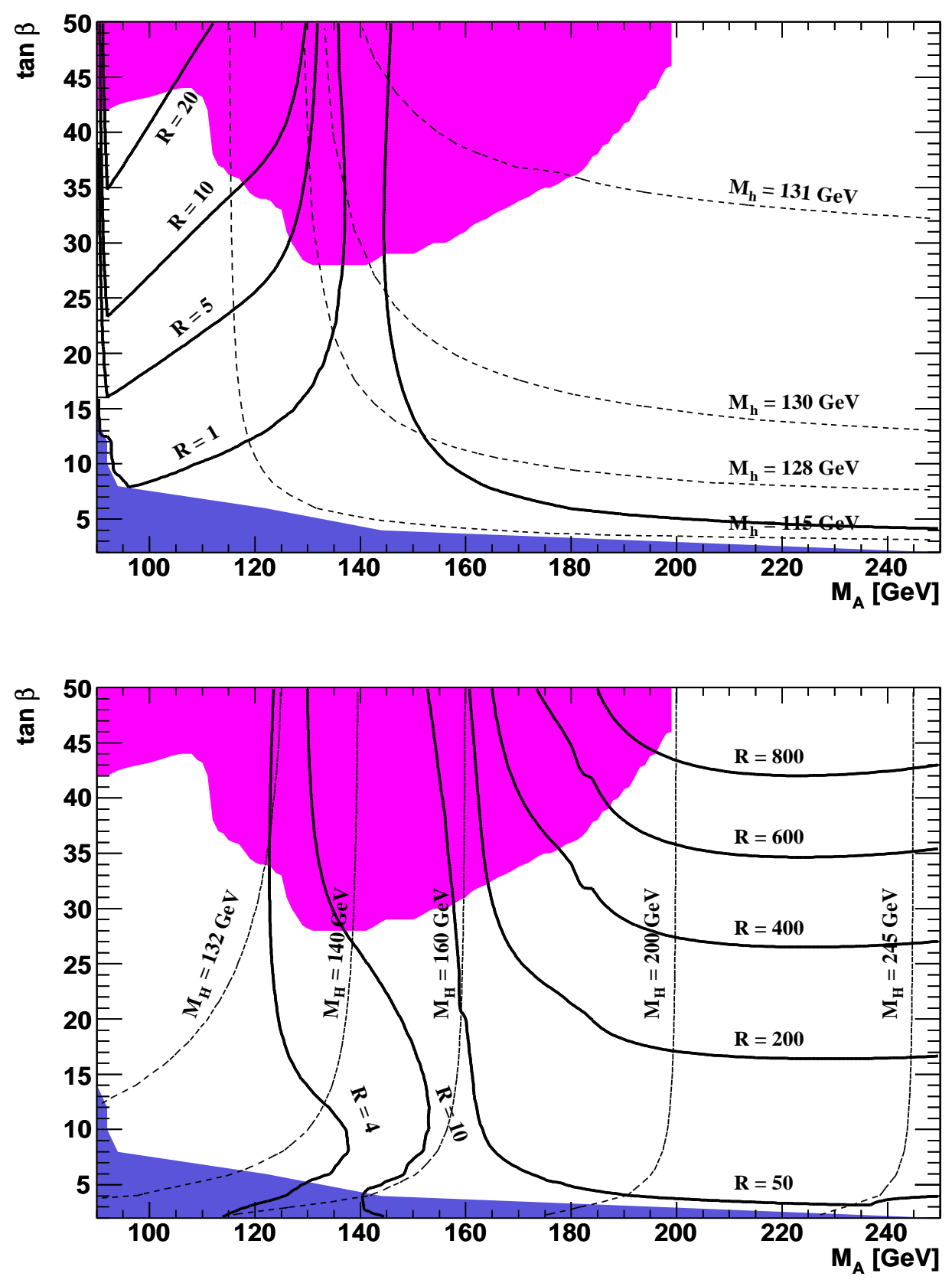

Figure 1: Contours for the ratio of signal events in the MSSM to those in the SM in the $h(H) \rightarrow b \bar{b}$ channel in CED production in the $M_{A}-\tan \beta$ plane are shown in the upper (lower) plot. The ratio is displayed in the $M_{h}^{\max }$ benchmark scenario with $\mu=+200 \mathrm{GeV}$. The values of the mass of the light (heavy) $\mathcal{C} \mathcal{P}$-even Higgs boson, $M_{h}\left(M_{H}\right)$, are indicated by contour lines. The dark shaded (blue) region corresponds to the parameter region that is excluded by the LEP Higgs searches, the lighter shaded (pink) areas are excluded by Tevatron Higgs searches. 
central values of the signal and background predictions discussed above, i.e., the remaining theoretical uncertainty in the signal-to-background ratios is not explicitly taken into account.

\section{Benchmark scenarios}

Due to the large number of MSSM parameters, a number of benchmark scenarios [42, 60] have been used for the interpretation of MSSM Higgs boson searches at LEP [61, 62] and at the Tevatron [63, 64]. Two of the most commonly used benchmark scenarios for the $\mathcal{C} \mathcal{P}_{-}$ conserving MSSM [42,60] were used in Ref. [14]:

- The $M_{h}^{\max }$ scenario:

In this scenario the parameters are chosen such that the mass of the light $\mathcal{C} \mathcal{P}$-even Higgs boson acquires its maximum possible values as a function of $\tan \beta$ (for fixed $M_{\mathrm{SUSY}}$, $m_{t}$, and $M_{A}$ set to its maximum value, $M_{A}=1 \mathrm{TeV}$ ). This was used in particular to obtain conservative $\tan \beta$ exclusion bounds [65] at LEP [62]. The parameters are:

$$
\begin{aligned}
& m_{t}=173.3 \mathrm{GeV}, \quad M_{\mathrm{SUSY}}=1 \mathrm{TeV}, \quad \mu=200 \mathrm{GeV}, \quad M_{2}=200 \mathrm{GeV}, \\
& X_{t}=2 M_{\mathrm{SUSY}} \quad A_{b}=A_{t}, \quad m_{\tilde{g}}=0.8 M_{\mathrm{SUSY}} .
\end{aligned}
$$

- The no-mixing scenario:

This benchmark scenario is the same as the $M_{h}^{\max }$ scenario, but with vanishing mixing in the $\tilde{t}$ sector and with a higher SUSY mass scale (the latter has been chosen to avoid conflict with the exclusion bounds from the LEP Higgs searches [61,62]),

$$
\begin{aligned}
& m_{t}=173.3 \mathrm{GeV}, \quad M_{\mathrm{SUSY}}=2 \mathrm{TeV}, \quad \mu=200 \mathrm{GeV}, \quad M_{2}=200 \mathrm{GeV}, \\
& X_{t}=0 \quad A_{b}=A_{t}, \quad m_{\tilde{g}}=0.8 M_{\mathrm{SUSY}} .
\end{aligned}
$$

As discussed above, in order to study the impact of potentially large corrections in the $b / \tilde{b}$ sector it is useful to vary the absolute value and sign of the parameter $\mu$. The other MSSM parameters that are not specified above have only a minor impact on MSSM Higgs-boson phenomenology. In our numerical analysis below we fix them such that all soft SUSY-breaking parameters in the diagonal entries of the sfermion mass matrices are set to $M_{\mathrm{SUSY}}$, and the trilinear couplings for all sfermions are set to $A_{t}$.

The benchmark scenarios defined above had been designed to facilitate the Higgs-boson searches at LEP, the Tevatron and the LHC. Consequently, they do not pay particular attention to the compliance with other constraints, such as electroweak precision observables, $B$ physics observables and the abundance of cold dark matter (CDM), see, for instance, Ref. [66] and references therein. In Ref. [67] four new MSSM benchmark scenarios based on the NUHM2 [68] were defined 3. In these scenarios the abundance of the lightest SUSY

\footnotetext{
3 The NUHM2, which stands for "Non-Universal Higgs Model type 2", assumes common mass scales for scalars, $m_{0}$, for fermions, $m_{1 / 2}$, and for trilinear couplings, $A_{0}$, at the Grand Unification scale, $M_{\mathrm{GUT}} \sim$ $2 \times 10^{16} \mathrm{GeV}$. However, the two scalar soft SUSY-breaking parameters of the Higgs sector are assumed to be unconnected to $m_{0}$. Together with $\tan \beta$ the NUHM2 is described by six free parameters (and the sign of $\mu$ ).
} 
particle, the lightest neutralino, in the early universe is compatible within the $M_{A}-\tan \beta$ plane with the CDM constraints as measured by WMAP [69]. The parameters chosen for the benchmark planes are also in agreement with electroweak precision and $B$-physics constraints, see Ref. [67] for further details. Below we will present the prospects for CED Higgs production also in two of these four "CDM benchmark scenarios", labeled P3 and P4. More details about their definition can be found in Ref. [67].

\section{Bounds from Higgs searches at LEP and the Tevatron}

The Higgs bosons of the SM and the MSSM have been searched for at LEP [61, 62] and the Tevatron [63, 64. The results presented in Ref. [14] showed the parts of the $M_{A}-\tan \beta$ planes that are excluded by the LEP Higgs-boson searches. Since then the Tevatron has considerably improved its reach for the SUSY Higgs bosons, especially for relatively low $M_{A}$ and large $\tan \beta$. The bounds obtained at the Tevatron (together with the previously known LEP bounds) have been implemented into the Fortran code HiggsBounds [41] (linked to FeynHiggs [36 39] to provide the relevant Higgs masses and couplings). For any parameter point provided to HiggsBounds the code determines whether it is excluded at the 95\% C.L. based on the published exclusion data. These excluded regions from LEP and the Tevatron are marked in the MSSM $\left(M_{h}^{\max }\right.$, no-mixing and CDM benchmark scenarios) and the SM4 plots shown below. We have used the version HiggsBounds 1.2.0 for our evaluations.

\subsection{Updated discovery reach for neutral $\mathcal{C P}$-even Higgs bosons in the $M_{h}^{\max }$ and no-mixing scenarios}

In this section we present the updated prospects for observing the neutral $\mathcal{C} \mathcal{P}$-even MSSM Higgs bosons in CED production. We display our results in the $M_{A}-\tan \beta$ planes for the $M_{h}^{\max }$ and no-mixing benchmark scenarios (the new results for the "CDM benchmark scenarios" can be found in the next subsection). As explained there, also shown in the plots are the parameter regions excluded by the LEP Higgs searches (as dark shaded (blue) areas) and Tevatron Higgs-boson searches (as lighter shaded (pink) areas) as obtained with HiggsBounds [41. Concerning the Tevatron exclusion bounds it should be noted that they largely rely on the searches in the channel $b \bar{b} \rightarrow h, H, A \rightarrow \tau^{+} \tau^{-}$. The SM cross section used [70] for the normalization within HiggsBounds is evaluated using the MRST2002 NNLO PDFs [71]. Using the updated version MSTW2008 [72] results in a reduction of the cross section by $\sim 20 \%$, which translates into weaker bounds on $\tan \beta$ by about $10 \%$.

For each point in the parameter space we have evaluated the relevant Higgs production cross section times the Higgs branching ratio corresponding to the decay mode under investigation. The Higgs-boson masses, the decay branching ratios and the effective couplings for the production cross sections have been calculated with the program FeynHiggs [36 40]. The resulting theoretical cross section has been multiplied by the experimental efficiencies taking into account detector acceptances, experimental cuts and triggers as discussed in Ref. [14]. The backgrounds have been estimated according to the update given in the previous subsection.

This procedure has been carried out for four different assumptions on the luminosity scenario, see Sect. 1, for which the $5 \sigma$ discovery contours and contours for $3 \sigma$ significances 
(see below) have been obtained.

We start our analysis with the production of the lighter $\mathcal{C P}$-even Higgs boson, $h$, and its decay into bottom quarks. As explained in Sect. 2.1, the $h b \bar{b}$ coupling can be significantly enhanced compared to the SM case in the region of relatively small $M_{A}$ and large $\tan \beta$ (while in the decoupling region, $M_{A} \gg M_{Z}$, the lighter $\mathcal{C} \mathcal{P}$-even Higgs boson of the MSSM behaves like the SM Higgs boson). Therefore, in parameter regions where the relevant couplings are enhanced compared to the SM case, CED production of the lighter $\mathcal{C} \mathcal{P}$-even Higgs boson of the MSSM with subsequent decay to $b \bar{b}$ yields a higher event rate.

In Fig. 2 we show the $5 \sigma$ discovery contours (upper plot) and contours of $3 \sigma$ statistical significance (lower plot) for the $h \rightarrow b \bar{b}$ channel in CED production in the $M_{A}$-tan $\beta$ plane of the MSSM within the $M_{h}^{\text {max }}$ benchmark scenario. The results are shown for assumed effective integrated luminosities (see text, combining ATLAS and CMS) of $60 \mathrm{fb}^{-1}, 60 \mathrm{fb}^{-1} \mathrm{eff} \times 2$, $600 \mathrm{fb}^{-1}$ and $600 \mathrm{fb}^{-1}$ eff $\times 2$. Since the lighter $\mathcal{C} \mathcal{P}$-even Higgs boson of the MSSM is likely to be detectable also in "conventional" Higgs search channels at the LHC (see for example Refs. [73, 74]), a $3 \sigma$ statistical significance for the CED channel could be considered as sufficient. The values of the mass of the light $\mathcal{C P}$-even Higgs boson, $M_{h}$, are indicated by contour lines. The dark shaded (blue) region corresponds to the parameter region that is excluded by the LEP Higgs searches, the lighter shaded (pink) areas are excluded by Tevatron Higgs searches.

The $5 \sigma$ discovery regions cover the parameter space of $M_{A} \leq 135 \mathrm{GeV}$ and $\tan \beta \gtrsim 10$, depending on the luminosity assumption. Some parts at larger $\tan \beta$ are excluded by the Tevatron Higgs-boson searches. The $3 \sigma$ regions are much larger and cover nearly the whole $M_{A}$-tan $\beta$ plane in the high-luminosity scenarios. The only exception is a funnel around $M_{A} \approx 140 \mathrm{GeV}$ for $\tan \beta<25$ or $\tan \beta>30$, where $\tan \beta \gtrsim 28$ in the uncovered region is excluded by the Tevatron Higgs searches. The fact that coverage up to highest $M_{A}$ values displayed in Fig. 2 is obtained in the high-luminosity scenarios indicates that in this case a $3 \sigma$ significance would be obtained for a SM Higgs with a mass value corresponding to the light $\mathcal{C P}$-even Higgs mass of the MSSM. In comparison with the results presented in Ref. [14 one can see that the various updates of the analysis enlarge the regions allowing a $5 \sigma$ discovery as well as the ones yielding a $3 \sigma$ significance. Similarly to the case of the ratios discussed in Sect. 2.2, the enhancements with respect to the previous results of Ref. [14] are of up to a factor of 2 for $M_{A} \lesssim 140 \mathrm{GeV}$, while they do not exceed $10 \%$ for $M_{A} \gtrsim 140 \mathrm{GeV}$.

The results look very similar in the no-mixing scenario as shown in Fig. 3. The $5 \sigma$ discovery regions, given in the upper plot, end at somewhat lower $M_{A}$ values, $M_{A} \lesssim 125 \mathrm{GeV}$. The uncovered funnel for the $3 \sigma$ significance, given in the lower plot has shrunk such that only $\tan \beta \lesssim 15$ and $\tan \beta \gtrsim 45$ remain uncovered. Concerning the experimental bounds, the LEP bounds extend to higher $\tan \beta$ values, reflecting the fact that much lower $M_{h}$ values are realized in this scenario 4 The Tevatron bounds, on the other hand, remain nearly unchanged. The comparison with the results in Ref. [14] leads to the same conclusions as in the $M_{h}^{\max }$ scenario: the various updates enlarge the regions delimited by the $5 \sigma$ discovery contours as well as by the $3 \sigma$ significance contours (with the details discussed in the case of the $M_{h}^{\max }$ scenario).

${ }^{4}$ The fact that the LEP bounds do not reach $M_{h}=114 \mathrm{GeV}$ [61,62 reflects the theory uncertainty of $\sim 3 \mathrm{GeV}$ in $M_{h}$ [38 taken into account for the LEP bounds. 

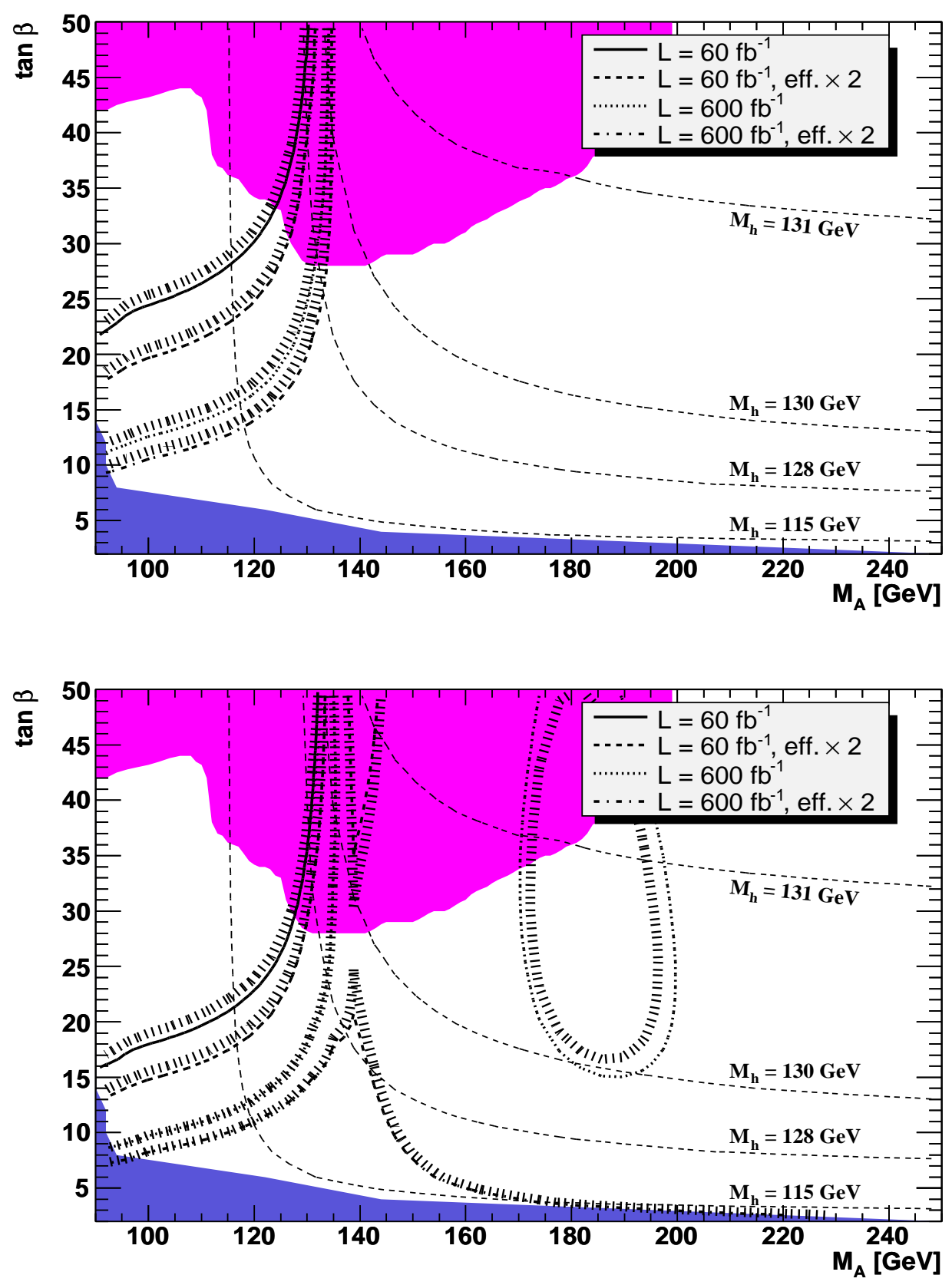

Figure 2: $5 \sigma$ discovery contours (upper plot) and contours of $3 \sigma$ statistical significance (lower plot) for the $h \rightarrow b \bar{b}$ channel in CED production in the $M_{A}-\tan \beta$ plane of the MSSM within the $M_{h}^{\max }$ benchmark scenario. The results are shown for assumed effective luminosities (see text, combining ATLAS and CMS) of $60 \mathrm{fb}^{-1}, 60 \mathrm{fb}^{-1} \mathrm{eff} \times 2,600 \mathrm{fb}^{-1}$ and $600 \mathrm{fb}^{-1} \mathrm{eff} \times 2$. The values of the mass of the light $\mathcal{C} \mathcal{P}$-even Higgs boson, $M_{h}$, are indicated by contour lines. The dark shaded (blue) region corresponds to the parameter region that is excluded by the LEP Higgs searches, the lighter shaded (pink) areas are excluded by Tevatron Higgs searches. 

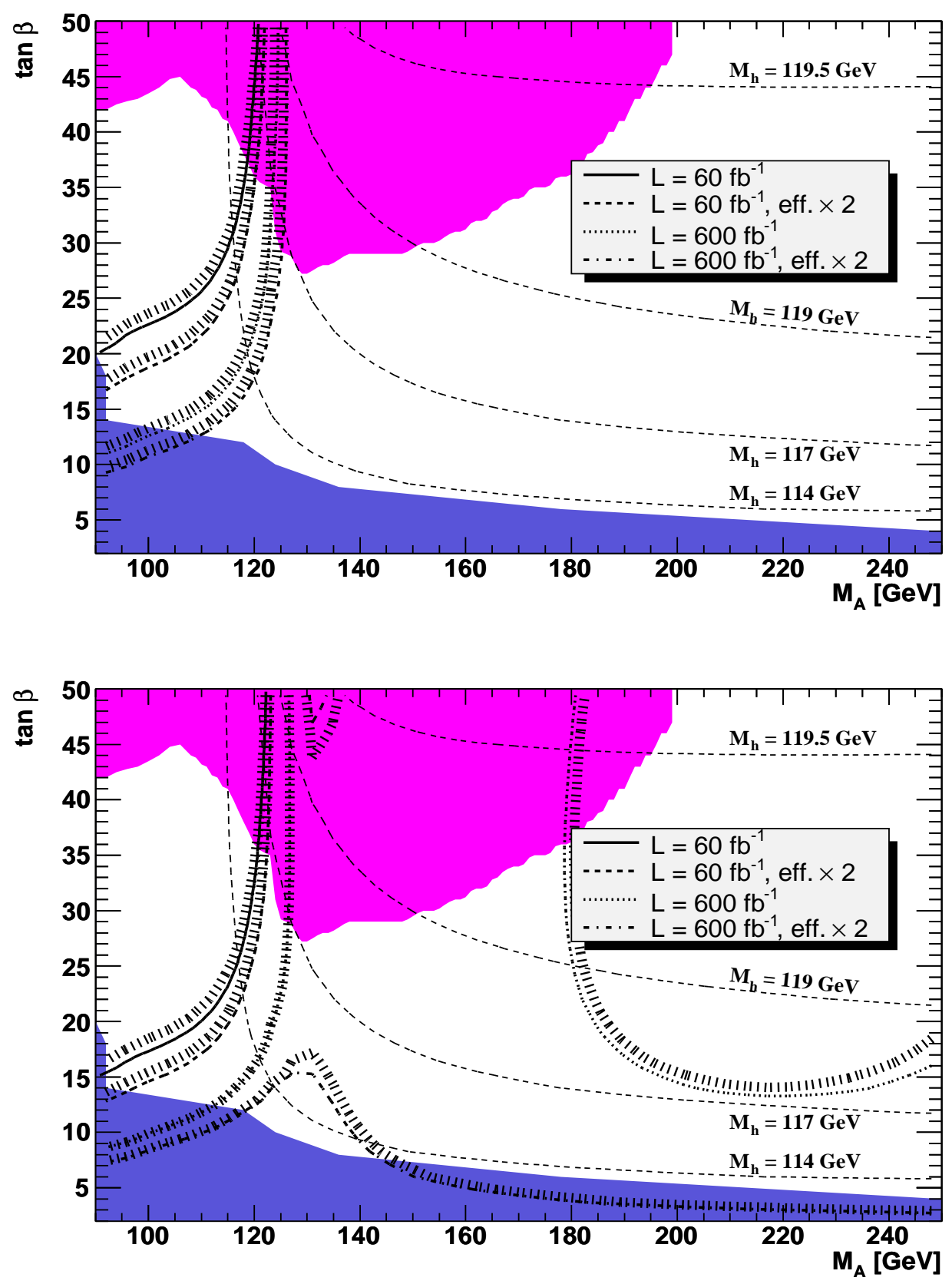

Figure 3: $5 \sigma$ discovery contours (upper plot) and contours of $3 \sigma$ statistical significance (lower plot) for the $h \rightarrow b \bar{b}$ channel in CED production in the $M_{A}-\tan \beta$ plane of the MSSM within the no-mixing benchmark scenario. The results are shown for assumed effective luminosities (see text, combining ATLAS and CMS) of $60 \mathrm{fb}^{-1}, 60 \mathrm{fb}^{-1}$ eff $\times 2,600 \mathrm{fb}^{-1}$ and $600 \mathrm{fb}^{-1}$ eff $\times 2$. The values of the mass of the light $\mathcal{C P}$-even Higgs boson, $M_{h}$, are indicated by contour lines. The dark shaded (blue) region corresponds to the parameter region that is excluded by the LEP Higgs searches, the lighter shaded (pink) areas are excluded by Tevatron Higgs searches. 
The CED production process with subsequent decay into bottom quarks is of particular relevance since this channel may be the only possibility for directly accessing the $h b \bar{b}$ coupling 5 although the decay into bottom quarks is by far the dominant decay mode of the lighter $\mathcal{C P}$-even Higgs boson in nearly the whole parameter space of the MSSM (and it is also the dominant decay of a light SM-like Higgs). For this reason information on the bottom Yukawa coupling is important for determining any Higgs-boson coupling at the LHC (rather than just ratios of couplings), see Refs. [28, 29, 76]. Figs. 2, 3 show that at the $3 \sigma$ level a significantly larger part of the $M_{A}-\tan \beta$ plane can be covered compared to the $5 \sigma$ discovery contours. In particular, in the " $600 \mathrm{fb}^{-1} \mathrm{eff} \times 2$ " scenario the coverage in both benchmark scenarios extends to large $M_{A}$ values and small values of $\tan \beta$. With the exception of a small parameter region around $M_{A} \lesssim 140(130) \mathrm{GeV}$ and low $\tan \beta$ values, in the $M_{h}^{\max }$ (no-mixing) scenario the whole $M_{A}-\tan \beta$ plane of the MSSM (and also the case of a light SM-like Higgs) can be covered with the CED process in this case. This important result implies that if the CED channel can be utilized at high instantaneous luminosity (which requires in particular that pile-up background is brought under control, see the discussion in Sect. 2.2 and in Ref. [14]) there is a good chance to detect the lighter $\mathcal{C P}$-even Higgs boson of the MSSM in this channel with subsequent decay into bottom quarks, yielding crucial information on the properties of the new state.

The updated prospects for the $h \rightarrow \tau^{+} \tau^{-}$channel in the $M_{h}^{\max }$ scenario are presented in Fig. 4. The $5 \sigma$ contours, shown in the upper plot, cover slightly smaller regions than the corresponding contours for the $h \rightarrow b \bar{b}$ channel in Fig. 2. The resulting features are similar for the $3 \sigma$ contours shown in the lower plot, with the exception of the high luminosity scenarios, where in contrast to the $h \rightarrow b \bar{b}$ channel the parameter region with $M_{A} \gtrsim 140 \mathrm{GeV}$ is left uncovered at the $3 \sigma$ level. This behaviour is a consequence of the fact that the sensitivity for a SM Higgs boson of the same mass drops below the $3 \sigma$ level when going from the $h \rightarrow b \bar{b}$ channel to the $\tau^{+} \tau^{-}$channel. The $5 \sigma$ contours delimit the areas between the corner of $M_{A} \lesssim 125 \mathrm{GeV}$ and $\tan \beta \gtrsim 35$ for the $60 \mathrm{fb}^{-1}$, and $M_{A} \lesssim 135 \mathrm{GeV}$ and $\tan \beta \gtrsim 12$ for the $600 \mathrm{fb}^{-1}$ eff $\times 2$ luminosity scenario. Correspondingly for the $3 \sigma$ contours, the corner for the $60 \mathrm{fb}^{-1}$ luminosity scenario extends up to $M_{A} \lesssim 130 \mathrm{GeV}$ and $\tan \beta \gtrsim 25$, while the area for the $600 \mathrm{fb}^{-1}$ eff $\times 2$ luminosity scenario extends down to $\tan \beta \gtrsim 9$. When compared to the results in Ref. [14] one can see that the regions covered by the new $5 \sigma$ and $3 \sigma$ contours are larger, but some parts are meanwhile excluded by the Tevatron Higgs boson searches (with the details given in the discussion of the $h \rightarrow b \bar{b}$ channel).

We now turn to the prospects for producing the heavier $\mathcal{C P}$-even Higgs boson of the MSSM in CED channels. The discovery reach in the "conventional" search channels at the LHC, in particular $b \bar{b} \rightarrow H / A \rightarrow \tau^{+} \tau^{-}$, covers the parameter region of high $\tan \beta$ and not too large $M_{A}$ [73, 74, 77, 78, while a "wedge region" [73, 74, 79] remains where the heavy MSSM Higgs bosons escape detection at the LHC (the discovery reach is somewhat extended if decays of the heavy MSSM Higgs bosons into supersymmetric particles can be utilized [73, 74]). CED production of the heavier $\mathcal{C}$ P-even Higgs boson of the MSSM with subsequent decay into bottom quarks provides a unique opportunity for accessing its bottom Yukawa coupling in a mass range where for a SM Higgs boson the decay rate into bottom quarks would be

\footnotetext{
${ }^{5}$ Another interesting idea to access the $b \bar{b}$ coupling to the Higgs boson is the production via Higgsstrahlung, $V^{*} \rightarrow V H\left(V=W^{ \pm}, Z\right)$ in a strongly boosted system [73, 75].
} 

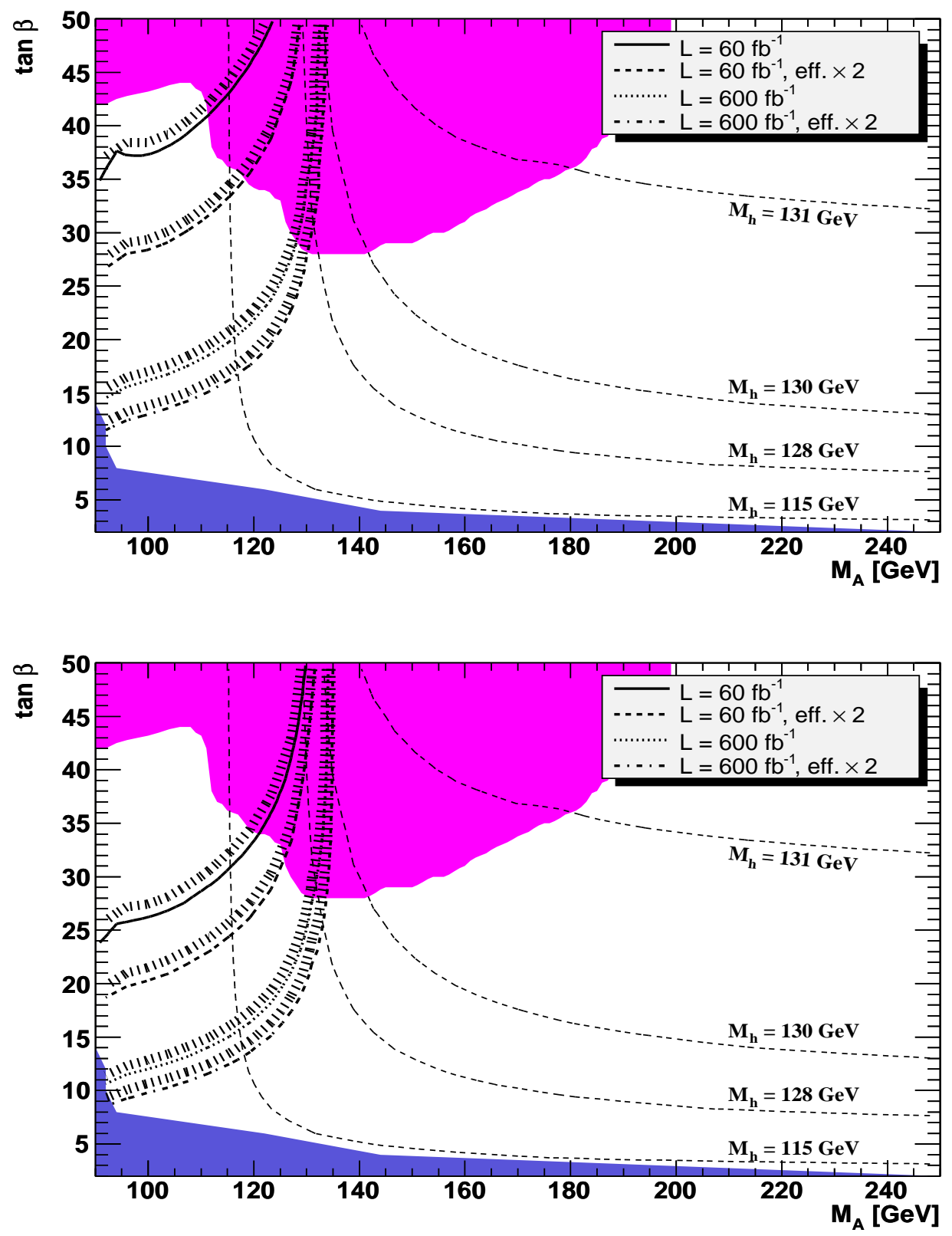

Figure 4: $5 \sigma$ discovery contours (upper plot) and contours of $3 \sigma$ statistical significance (lower plot) for the $h \rightarrow \tau^{+} \tau^{-}$channel in CED production in the $M_{A}$-tan $\beta$ plane of the MSSM within the $M_{h}^{\max }$ benchmark scenario. The results are shown for assumed effective luminosities (see text, combining ATLAS and CMS) of $60 \mathrm{fb}^{-1}, 60 \mathrm{fb}^{-1} \mathrm{eff} \times 2,600 \mathrm{fb}^{-1}$ and $600 \mathrm{fb}^{-1} \mathrm{eff} \times 2$. The values of the mass of the light $\mathcal{C} \mathcal{P}$-even Higgs boson, $M_{h}$, are indicated by contour lines. The dark shaded (blue) region corresponds to the parameter region that is excluded by the LEP Higgs searches, the lighter shaded (pink) areas are excluded by Tevatron Higgs searches. 
negligibly small.

It is well known that the properties of the heavier $\mathcal{C} \mathcal{P}$-even Higgs boson of the MSSM differ very significantly from the ones of a SM Higgs in the region where $M_{H}, M_{A} \gtrsim 150 \mathrm{GeV}$. While for a SM Higgs the $\mathrm{BR}(H \rightarrow b \bar{b})$ is strongly suppressed in this mass region, the decay into bottom quarks is the dominant decay mode for the heavier $\mathcal{C P}$-even MSSM Higgs boson (as long as no decays into supersymmetric particles or lighter Higgs bosons are open). In Ref. 14 it was shown that the MSSM process in the $H \rightarrow b \bar{b}$ channel is significantly enhanced compared to the SM case essentially everywhere in the unexcluded part of the $M_{A}-\tan \beta$ plane. Here also the $\Delta_{b}$ corrections play a significant role [60. While in the case of positive $\mu$ the higher-order contribution $\Delta_{b}$ yields a suppression of the bottom Yukawa coupling, the opposite effect occurs if the parameter $\mu$ is negative.

In Figs. 50 we show the contours of $5 \sigma$ (upper plots) and $3 \sigma$ (lower plots) statistical significances obtained in the four luminosity scenarios specified above for the $H \rightarrow b \bar{b}$ channel in CED production within the $M_{h}^{\max }$ scenario for $\mu=+200 \mathrm{GeV}$, the $M_{h}^{\max }$ scenario for $\mu=-500 \mathrm{GeV}$ and the no-mixing scenario for $\mu=+200 \mathrm{GeV}$, respectively. According to our brief discussion above (see Refs. [14,60] for more details) the following general pattern of the $5 \sigma$ and $3 \sigma$ contours is found: the coverage in the $M_{A}-\tan \beta$ plane is largest in the $M_{h}^{\max }$ scenario for $\mu=-500 \mathrm{GeV}$ (Fig. 6), followed by the no-mixing scenario for $\mu=$ $+200 \mathrm{GeV}$ (Fig. (7) and the $M_{h}^{\max }$ scenario for $\mu=+200 \mathrm{GeV}$ (Fig. 55). In the case of the lowest luminosity scenario, $60 \mathrm{fb}^{-1}$, the areas given by the $5 \sigma$ contours are largely ruled out by Tevatron Higgs-boson searches, except for the case of $\mu=-500 \mathrm{GeV}$. However, at the $3 \sigma$ level for $\mu=-500 \mathrm{GeV}$ (Fig. 6) and in the no-mixing scenario (Fig. 7) CED production of the heavy $\mathcal{C} \mathcal{P}$-even Higgs with $60 \mathrm{fb}^{-1}$ would cover substantial parts of the MSSM parameter space that are unexcluded by the Higgs searches carried out so far at the Tevatron (and elsewhere).

The high-luminosity scenarios could allow a $5 \sigma$ significance for $M_{H} \lesssim 240 \mathrm{GeV}$ for large $\tan \beta$ in the $M_{h}^{\max }$ scenario with $\mu=+200 \mathrm{GeV}$. This is extended to $M_{H} \lesssim 290 \mathrm{GeV}$ for $\mu=-500 \mathrm{GeV}$, and reaches $M_{H} \lesssim 255 \mathrm{GeV}$ in the no-mixing scenario, again with $\mu=+200 \mathrm{GeV}$. The $3 \sigma$ contours extend the reach by up to $\sim 20 \mathrm{GeV}$ in $M_{H}$. At low $\tan \beta$ the $3 \sigma$ reach goes down to (i.e. crosses the LEP exclusion bound) $M_{H} \sim 140-160 \mathrm{GeV}$, depending on the scenario.

We also note that the region left uncovered at the $3 \sigma$ level in the light $\mathcal{C P}$-even Higgsboson analyses can to a large extent be covered by searches for the heavy $\mathcal{C} \mathcal{P}$-even Higgs boson if the CED channel can be utilized at high instantaneous luminosity. Thus, a combined $h$ and $H$-analysis in this case would yield a reasonable signal of CED Higgs-boson production at the $3 \sigma$ level in nearly the whole parameter space that is unexcluded by the LEP and Tevatron searches.

In comparison with Ref. [14] one can see that the various updates (see Sect. 2.2), as in the case of the light $\mathcal{C P}$-even Higgs boson, lead to a somewhat larger coverage in the $M_{A}$-tan $\beta$ plane. More specifically, on average, the results are enhanced by a factor of $\sim 1.6$ in the regions where old significances are larger than 1 . In the rest of the parameter space they do not exceed 2.1 .

To summarize, the CED production at the LHC may be a unique way to access the bottom Yukawa coupling of a Higgs boson as heavy as $290 \mathrm{GeV}$ (which would obviously be a clear sign of physics beyond the Standard Model). Within the MSSM, however, this channel 
does not cover additional parameter space of the so-called "LHC wedge" (see the discussion in Ref. [14]).

We finish the update of the CED reach in the conventional benchmark scenarios with the $H \rightarrow \tau^{+} \tau^{-}$channel in the $M_{h}^{\text {max }}$ scenario as shown in Fig. 8. The $5 \sigma$ discovery regions shown in the upper plot are only visible for the high-luminosity scenarios, (apart from a small wedge around $M_{A}=140 \mathrm{GeV}$ and $\tan \beta \gtrsim 43$, where also lower luminosity could yield $5 \sigma$ ). However, they are excluded to a large extent by the Tevatron Higgs-boson searches. In the 600 $\mathrm{fb}^{-1}$ eff $\times 2$ scenario, $5 \sigma$ coverage is reached for $\tan \beta \sim 15-30$ and for $M_{A}=110-180 \mathrm{GeV}$. The $3 \sigma$ areas, shown in the lower plot, extend substantially outside the parameter space probed by the Tevatron in the high luminosity scenarios. They cover up to $M_{H} \lesssim 220 \mathrm{GeV}$ for large $\tan \beta$. As for the $H \rightarrow b \bar{b}$ channel, the reach is larger than in the analysis of Ref. [14]. More specifically, on average, the results are enhanced by a factor of $\sim 1.4$ in the regions where old significances are larger than 1 . In the rest of the parameter space they do not exceed 1.6.

\subsection{Discovery reach for neutral $\mathcal{C} \mathcal{P}$-even Higgs bosons in the "CDM benchmark scenarios"}

In this section we present for the first time a detailed study of the prospects for observing the neutral $\mathcal{C P}$-even MSSM Higgs bosons in CED production in the "CDM benchmark scenarios" P3 and P4, see Sect. 2.2 (see also Ref. [17]). As before the results are displayed in $M_{A^{-}}$ $\tan \beta$ planes, where P3 (P4) is defined for values below $\tan \beta=40$ (50) and for $M_{A} \geq$ $100 \mathrm{GeV}$. As in the previous subsection, the plots also show contour lines for $M_{h}, M_{H}$ as well as the parameter regions excluded by the LEP Higgs searches (as dark shaded (blue) areas) and Tevatron Higgs-boson searches (as lighter shaded (pink) areas) as obtained with HiggsBounds [41]. It should be kept in mind that large parts of these planes are also in agreement with electroweak precision data and $B$ physics data 67. In this sense these scenarios fulfill all external constraints, contrary to the conventional $M_{h}^{\max }$ and no-mixing benchmark scenarios (which were designed to highlight specific characteristics of the MSSM Higgs sector).

The $5 \sigma$ discovery contours as well as the contours for $3 \sigma$ significances have been obtained in the same way as for the conventional benchmark scenarios. In general they show similar qualitative features as the results in the $M_{h}^{\max }$ and the no-mixing scenario.

The results for the channel $h \rightarrow b \bar{b}$ in P3 are presented in Fig. 9. The upper and lower plots show the contours for $5 \sigma$ discovery and $3 \sigma$ significance, respectively. A $5 \sigma$ discovery is possible for $M_{A} \lesssim 125 \mathrm{GeV}$ and moderate to large values of $\tan \beta$, i.e. $\tan \beta \gtrsim 10$, depending on the luminosity scenario. At the $3 \sigma$ level, the coverage extends to the rest of the plane in the $600 \mathrm{fb}^{-1}$ eff $\times 2$ scenario, leaving only a small uncovered funnel around $M_{A} \approx 125 \mathrm{GeV}$ and $\tan \beta \lesssim 15$ or $\tan \beta \gtrsim 42$. The LEP bounds exclude a region at $\tan \beta \lesssim 11$ and $M_{A} \lesssim 140 \mathrm{GeV}$ that is barely touched by the $5 \sigma$ discovery contours. However, the LEP searches exclude a large part of the funnel at small $\tan \beta$ that is left uncovered at the $3 \sigma$ level by CED Higgs searches in the $600 \mathrm{fb}^{-1}$ eff $\times 2$ scenario. Thus, the LEP exclusion regions are complementary to the parameter space covered by CED Higgs-boson production in P3. (As mentioned above, the fact that the LEP bounds do not reach $M_{h}=114 \mathrm{GeV}$ 61, 62, reflects the theory uncertainty of $\sim 3 \mathrm{GeV}$ in $M_{h}$ [38] taken into account for the LEP bounds.) 

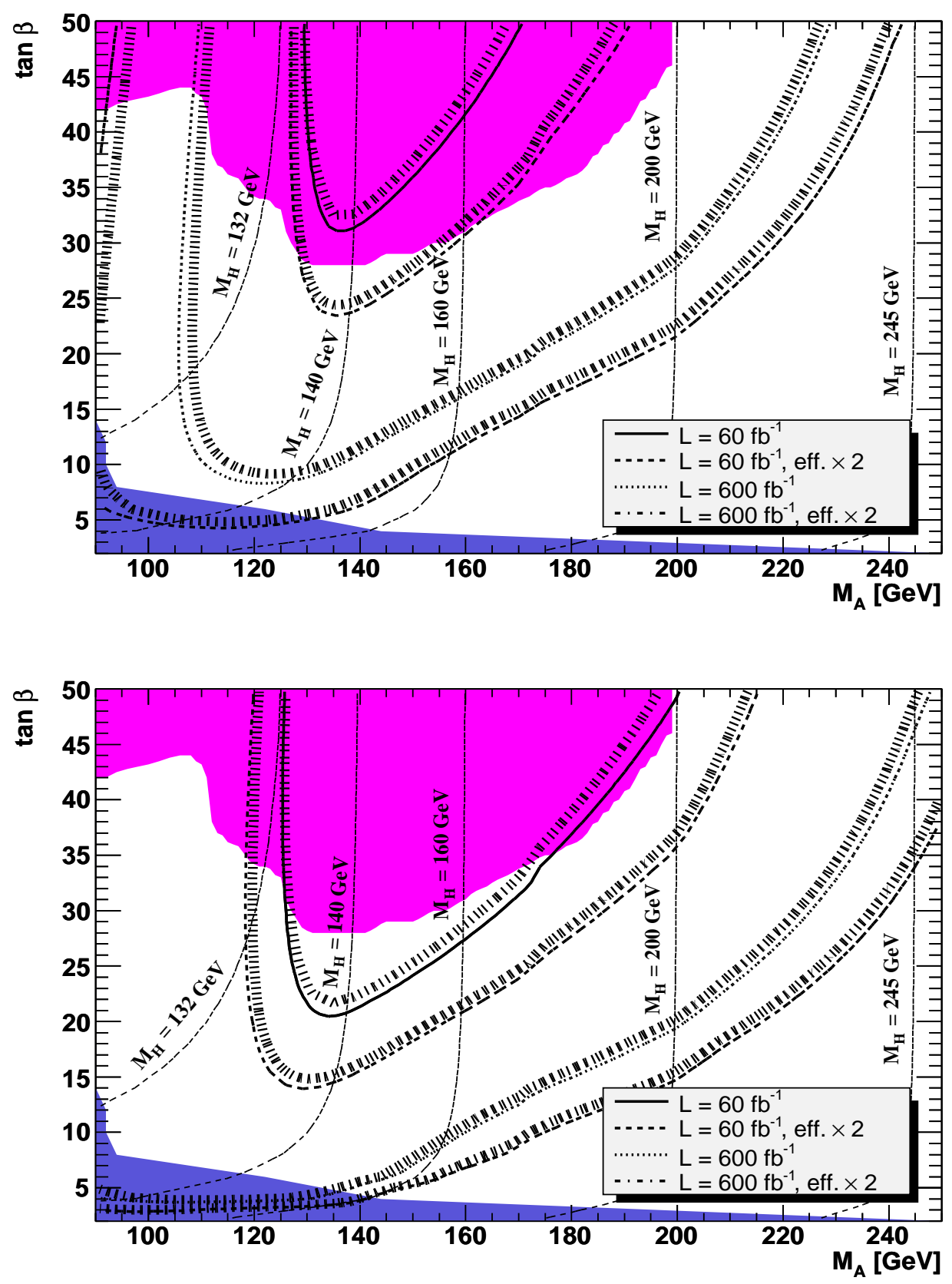

Figure 5: $5 \sigma$ discovery contours (upper plot) and contours of $3 \sigma$ statistical significance (lower plot) for the $H \rightarrow b \bar{b}$ channel in CED production in the $M_{A}$-tan $\beta$ plane of the MSSM within the $M_{h}^{\max }$ benchmark scenario. The results are shown for assumed effective luminosities (see text, combining ATLAS and CMS) of $60 \mathrm{fb}^{-1}, 60 \mathrm{fb}^{-1} \mathrm{eff} \times 2,600 \mathrm{fb}^{-1}$ and $600 \mathrm{fb}^{-1} \mathrm{eff} \times 2$. The values of $M_{H}$ are indicated by contour lines. The dark shaded (blue) region corresponds to the parameter region that is excluded by the LEP Higgs searches, the lighter shaded (pink) areas are excluded by Tevatron Higgs searches. 

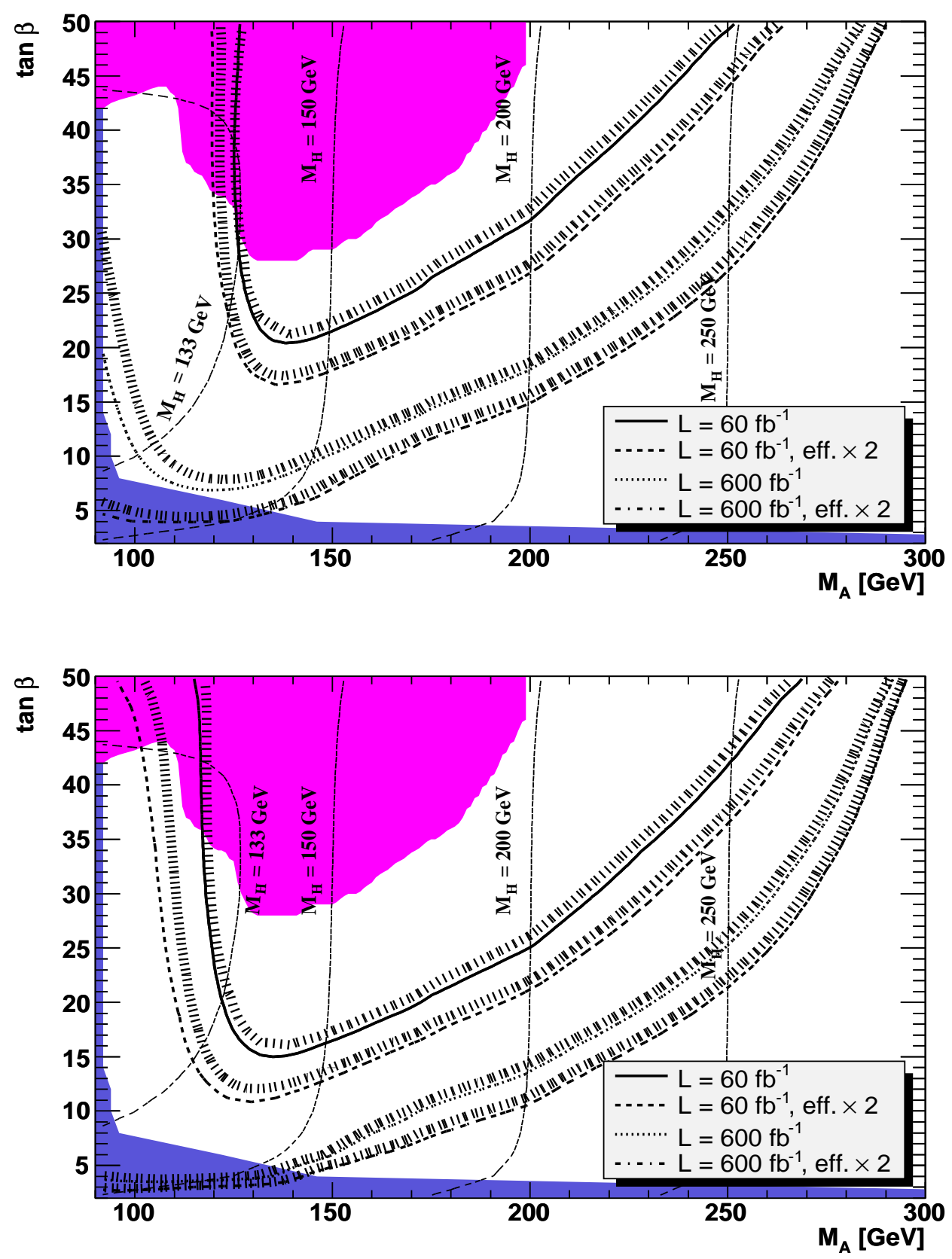

Figure 6: $5 \sigma$ discovery contours (upper plot) and contours of $3 \sigma$ statistical significance (lower plot) for the $H \rightarrow b \bar{b}$ channel in CED production in the $M_{A}-\tan \beta$ plane of the MSSM within the $M_{h}^{\max }$ benchmark scenario, but with $\mu=-500 \mathrm{GeV}$. The results are shown for assumed effective luminosities (see text, combining ATLAS and CMS) of $60 \mathrm{fb}^{-1}, 60$ $\mathrm{fb}^{-1}$ eff $\times 2,600 \mathrm{fb}^{-1}$ and $600 \mathrm{fb}^{-1}$ eff $\times 2$. The values of $M_{H}$ are indicated by contour lines. The dark shaded (blue) region corresponds to the parameter region that is excluded by the LEP Higgs searches, the lighter shaded (pink) areas are excluded by Tevatron Higgs searches. 

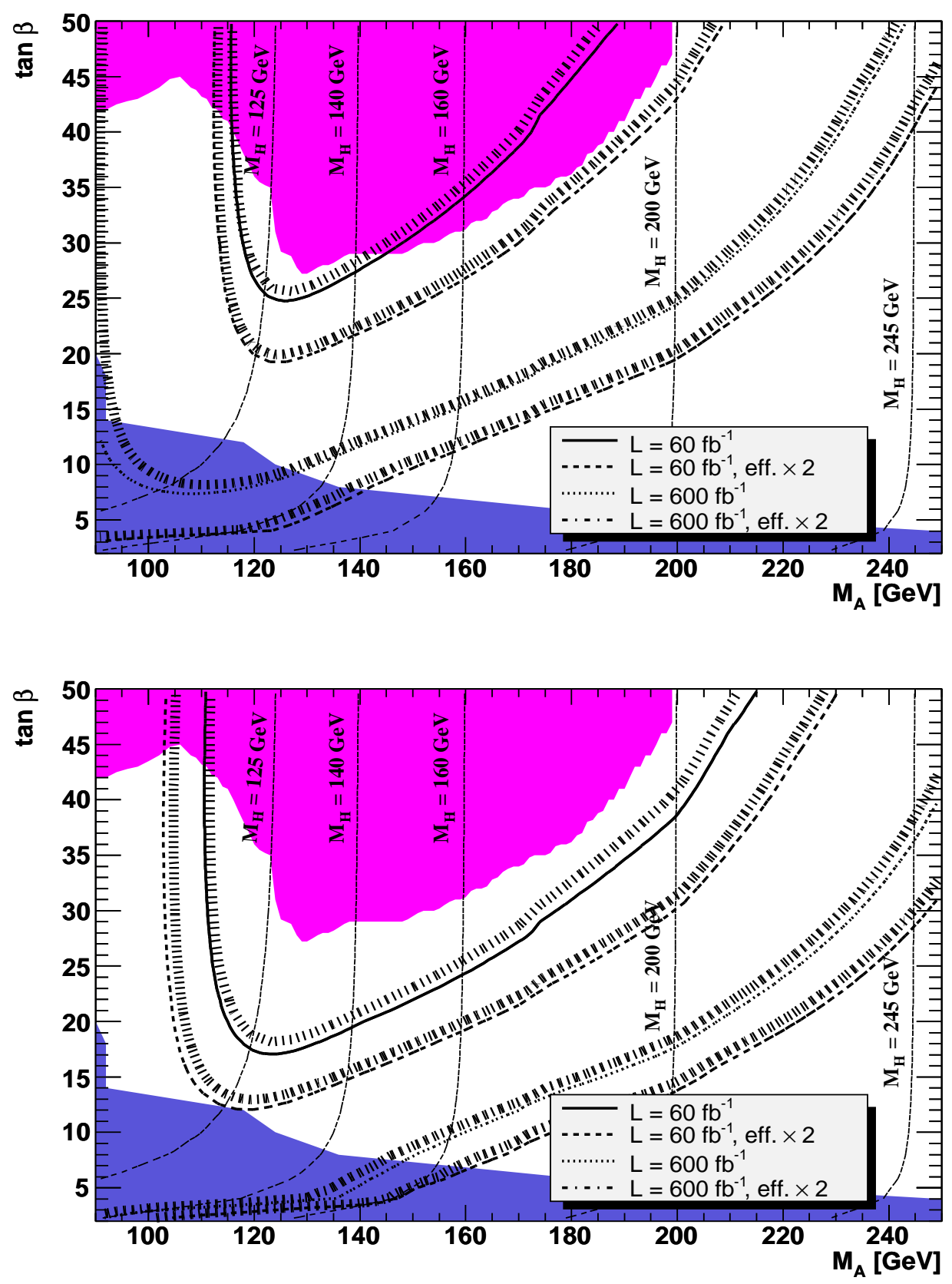

Figure 7: $5 \sigma$ discovery contours (upper plot) and contours of $3 \sigma$ statistical significance (lower plot) for the $H \rightarrow b \bar{b}$ channel in CED production in the $M_{A}-\tan \beta$ plane of the MSSM within the no-mixing benchmark scenario. The results are shown for assumed effective luminosities (see text, combining ATLAS and CMS) of $60 \mathrm{fb}^{-1}, 60 \mathrm{fb}^{-1}$ eff $\times 2,600 \mathrm{fb}^{-1}$ and $600 \mathrm{fb}^{-1}$ eff $\times 2$. The values of $M_{H}$ are indicated by contour lines. The dark shaded (blue) region corresponds to the parameter region that is excluded by the LEP Higgs searches, the lighter shaded (pink) areas are excluded by Tevatron Higgs searches. 

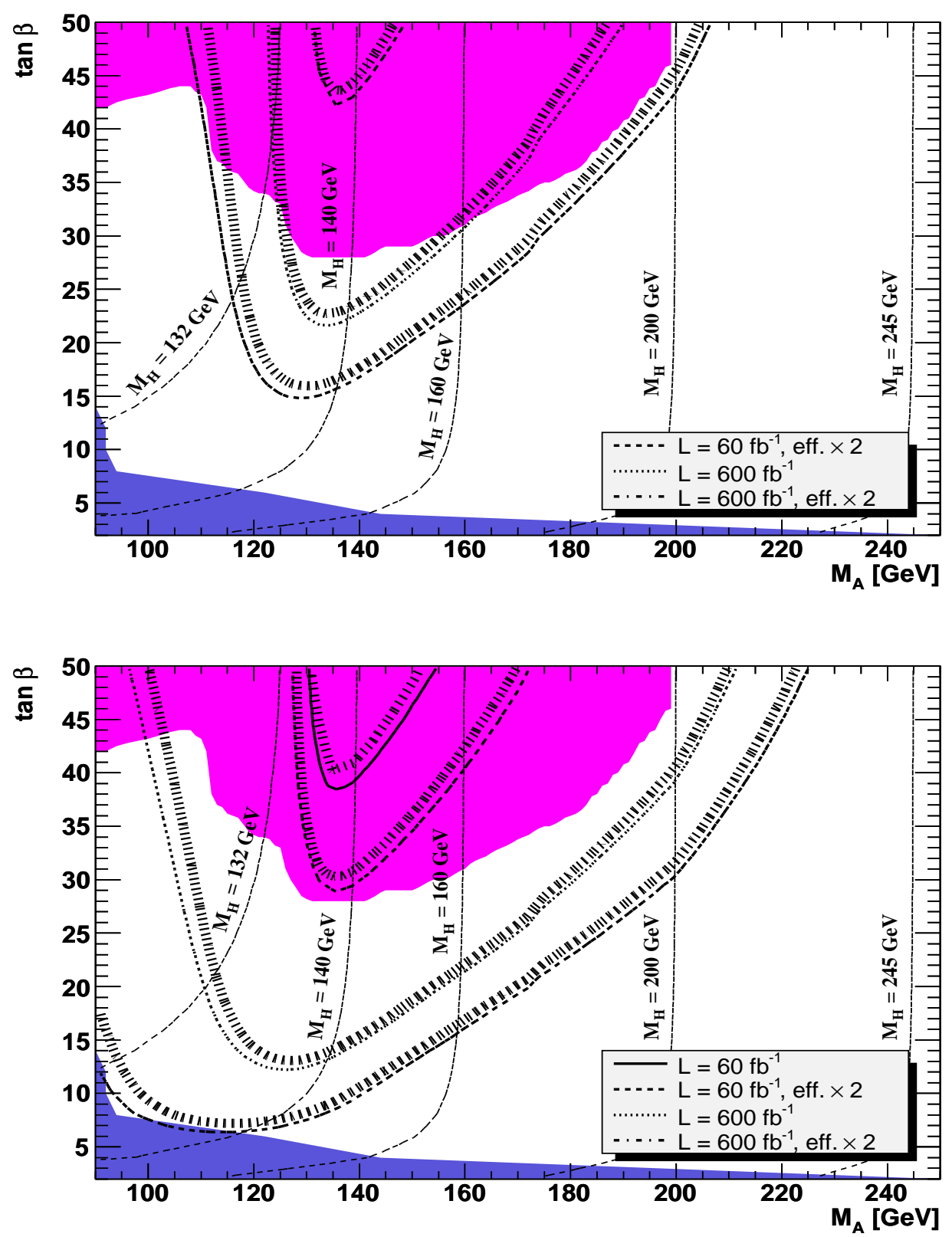

Figure 8: $5 \sigma$ discovery contours (upper plot) and contours of $3 \sigma$ statistical significance (lower plot) for the $H \rightarrow \tau^{+} \tau^{-}$channel in CED production in the $M_{A}$-tan $\beta$ plane of the MSSM within the $M_{h}^{\max }$ benchmark scenario. The results are shown for assumed effective luminosities (see text, combining ATLAS and CMS) of $60 \mathrm{fb}^{-1}, 60 \mathrm{fb}^{-1} \mathrm{eff} \times 2,600 \mathrm{fb}^{-1}$ and $600 \mathrm{fb}^{-1} \mathrm{eff} \times 2$. The values of $M_{H}$ are indicated by contour lines. The dark shaded (blue) region corresponds to the parameter region that is excluded by the LEP Higgs searches, the lighter shaded (pink) areas are excluded by Tevatron Higgs searches. 
The Tevatron searches exclude a parameter space with $\tan \beta \gtrsim 28$ around $M_{A}=140 \mathrm{GeV}$. Consequently, the uncovered funnel at $\tan \beta \gtrsim 42$ for CED Higgs-boson production in the $600 \mathrm{fb}^{-1}$ eff $\times 2$ scenario lies within the parameter region that is excluded by the Tevatron Higgs searches.

In Fig. 10 we present the corresponding results in the $h \rightarrow b \bar{b}$ channel for $\mathbf{P} 4$, which, as explained above, extends only up to $\tan \beta=40$. The $5 \sigma$ and $3 \sigma$ reach is similar to that in P3. However, due to the lower $M_{h}$ values realized in this scenario the LEP searches exclude an even larger part of the funnel at small $\tan \beta$ that is left uncovered at the $3 \sigma$ level by CED Higgs searches in the $600 \mathrm{fb}^{-1}$ eff $\times 2$ scenario.

In Fig. 11 we show the results for the $h \rightarrow \tau^{+} \tau^{-}$channel in the scenario P3. For the high luminosity cases a $5 \sigma$ coverage is obtained for $\tan \beta \gtrsim 15$ and $M_{A} \lesssim 120 \mathrm{GeV}$. A slightly larger region is covered at the $3 \sigma$ level, extending down to $\tan \beta \gtrsim 10$ in the high luminosity scenarios. Compared with the $M_{h}^{\text {max }}$ scenario, the coverage in $M_{A}$ is smaller by $\sim 10 \mathrm{GeV}$.

Now we turn to the analysis for the heavy $\mathcal{C P}$-even Higgs boson. In Fig. 12 we present the results for the channel $H \rightarrow b \bar{b}$ in the scenario P3. The reach is somewhat larger than in the $M_{h}^{\max }$ scenario and similar to the no-mixing scenario. $5 \sigma$ can be reached up to $M_{H} \lesssim 260 \mathrm{GeV}$ at large $\tan \beta$ and high luminosity. At low luminosity the region extends only up to $M_{H} \lesssim 210 \mathrm{GeV}$, and it is largely excluded by the Tevatron searches. The $3 \sigma$ significance contours move to larger $M_{H}$ values by $20-30 \mathrm{GeV}$. As above, the region left uncovered at the $3 \sigma$ level by CED light Higgs searches in the $600 \mathrm{fb}^{-1} \mathrm{eff} \times 2$ scenario is accessible via $\mathrm{CED}$ production of the heavy $\mathcal{C} \mathcal{P}$-even Higgs boson.

The corresponding results in the scenario $\mathbf{P} 4$ are presented in Fig. 13, The reach is similar to P3 (but restricted by construction to $\tan \beta \leq 40$ ). Again, a large fraction of the parameter space covered at $5 \sigma$ with low luminosity is excluded by the Tevatron searches, except for a region with $M_{A} \lesssim 135 \mathrm{GeV}$. The $3 \sigma$ significance contours, on the other hand, cover a large part of the unexcluded region between the Tevatron and the LEP limits even for the low-luminosity scenarios. In the high-luminosity scenario at $\tan \beta \lesssim 40$ we find that $M_{H}=240(260) \mathrm{GeV}$ can be covered at $5(3) \sigma$.

Finally we present the results for $H \rightarrow \tau^{+} \tau^{-}$for the scenario P3 in Fig. 14. The reach is slightly larger than in the $M_{h}^{\max }$ scenario as shown in Fig. 8. However, as in the conventional benchmark scenario the regions covered at $5 \sigma$ with low luminosity are excluded by the Tevatron searches. The coverage in the $600 \mathrm{fb}^{-1}$ and $600 \mathrm{fb}^{-1} \mathrm{eff} \times 2$ luminosity scenarios extends down to $\tan \beta \lesssim 15$ at $M_{A} \approx M_{H} \approx 120 \mathrm{GeV}$, and at $\tan \beta=50$ to $M_{A} \lesssim 210 \mathrm{GeV}$. The $3 \sigma$ coverage at the highest luminosity goes up to $M_{A} \approx 235 \mathrm{GeV}$ at $\tan \beta=50$ and down to $M_{A} \approx 135 \mathrm{GeV}$ around $\tan \beta=10$, where the coverage of the CED channel overlaps with the LEP exclusion bound.

To summarize, from the investigation of the conventional scenarios and the "CDM benchmark" scenarios we can draw the conclusion that there are promising prospects for the CED production of the light and / or heavy $\mathcal{C P}$-even Higgs bosons of the MSSM with subsequent decay to $b \bar{b}$ (and possibly to $\tau^{+} \tau^{-}$). The fact that we find similar results in the conventional benchmark scenarios and in the "CDM benchmark" scenarios strengthens the overall validity of our findings. While in the $60 \mathrm{fb}^{-1}$ and $60 \mathrm{fb}^{-1}$ eff $\times 2$ scenarios the results from the Tevatron Higgs searches meanwhile exclude some of the $5 \sigma$ regions that are accessible for the heavy $\mathcal{C} \mathcal{P}$-even Higgs in $\mathrm{CED}$ production, a significant coverage of currently unexplored parameter space can be achieved at the $3 \sigma$ level. If the CED channel can be utilized at high 

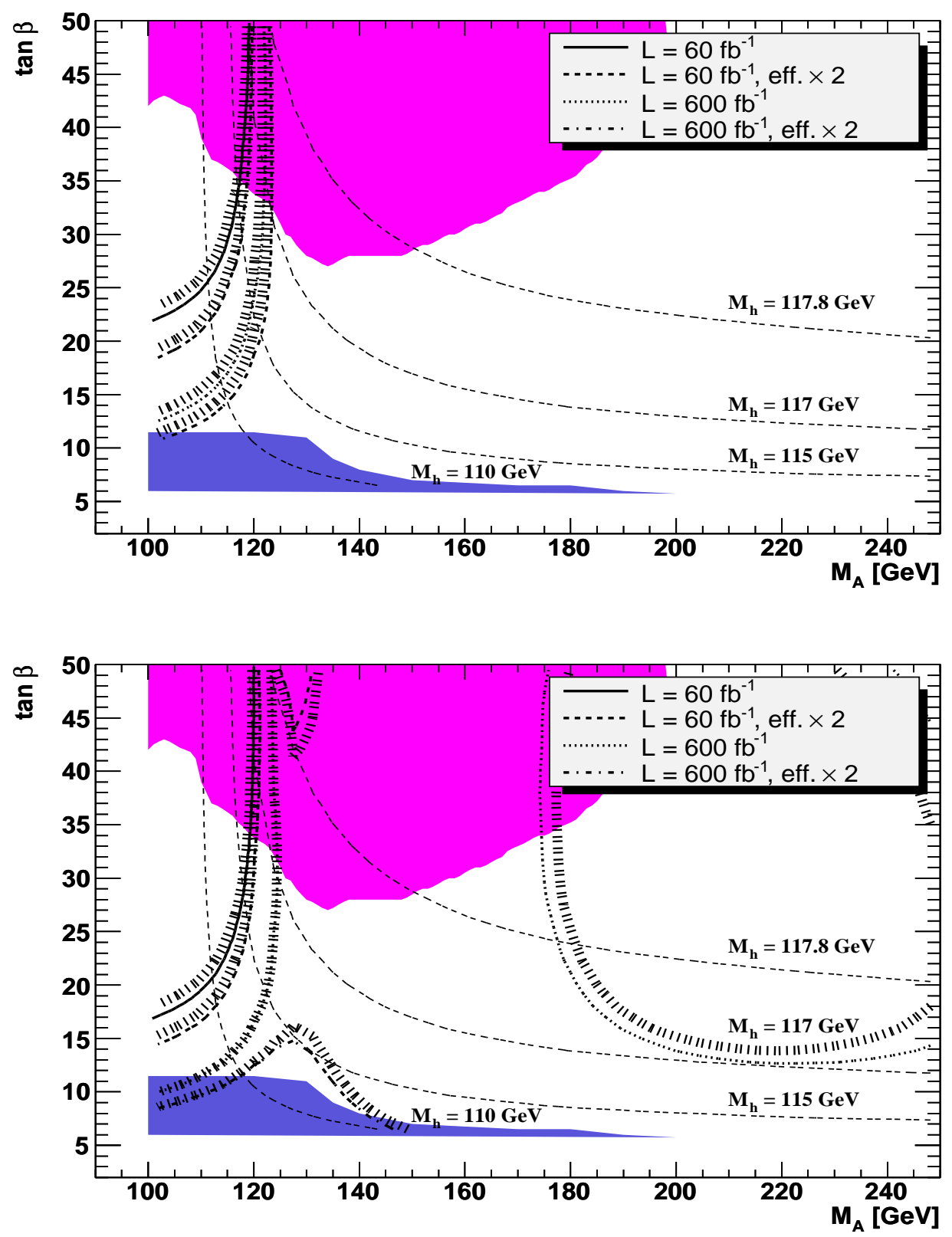

Figure 9: $5 \sigma$ discovery contours (upper plot) and contours of $3 \sigma$ statistical significance (lower plot) for the $h \rightarrow b \bar{b}$ channel in CED production in the $M_{A}-\tan \beta$ plane of the MSSM within the "CDM benchmark" scenario P3. The results are shown for assumed effective luminosities (see text, combining ATLAS and CMS) of $60 \mathrm{fb}^{-1}, 60 \mathrm{fb}^{-1}$ eff $\times 2,600 \mathrm{fb}^{-1}$ and $600 \mathrm{fb}^{-1} \mathrm{eff} \times 2$. The values of $M_{h}$ are indicated by contour lines. The dark shaded (blue) region corresponds to the parameter region that is excluded by the LEP Higgs searches, the lighter shaded (pink) areas are excluded by Tevatron Higgs searches. 

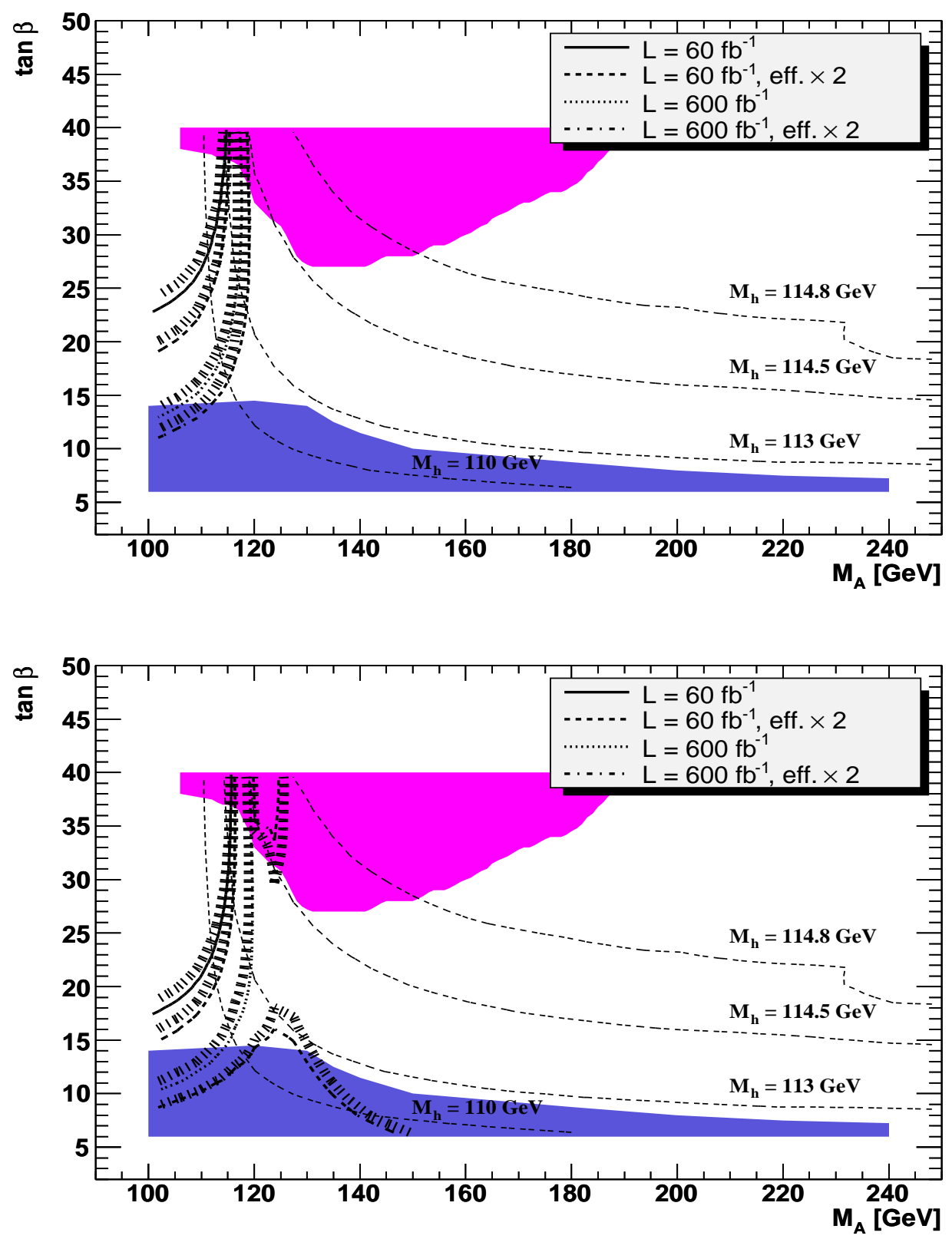

Figure 10: $5 \sigma$ discovery contours (upper plot) and contours of $3 \sigma$ statistical significance (lower plot) for the $h \rightarrow b \bar{b}$ channel in CED production in the $M_{A}-\tan \beta$ plane of the MSSM within the "CDM benchmark" scenario P4. The results are shown for assumed effective luminosities (see text, combining ATLAS and CMS) of $60 \mathrm{fb}^{-1}, 60 \mathrm{fb}^{-1}$ eff $\times 2,600 \mathrm{fb}^{-1}$ and $600 \mathrm{fb}^{-1} \mathrm{eff} \times 2$. The values of $M_{h}$ are indicated by contour lines. The dark shaded (blue) region corresponds to the parameter region that is excluded by the LEP Higgs searches, the lighter shaded (pink) areas are excluded by Tevatron Higgs searches. 

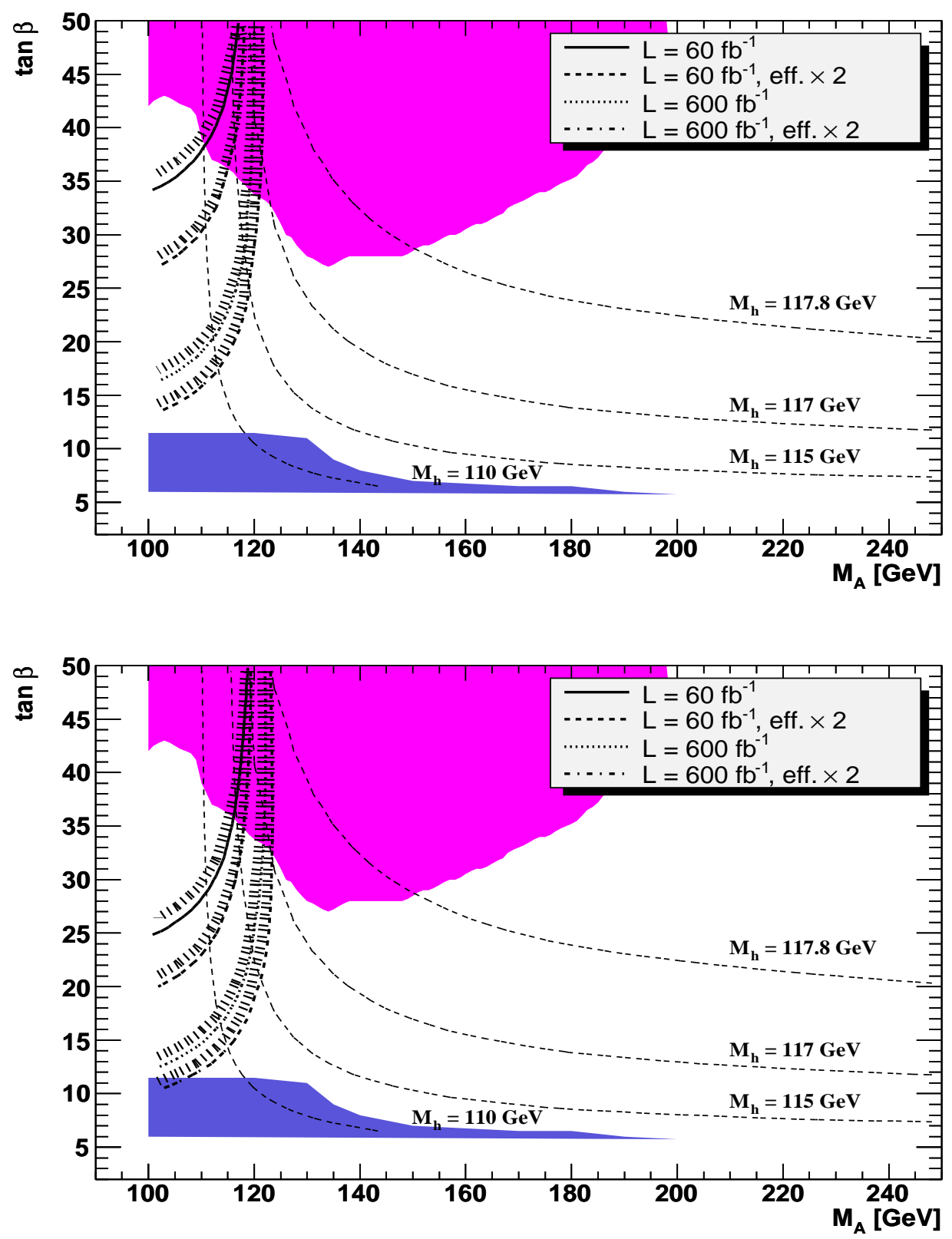

Figure 11: $5 \sigma$ discovery contours (upper plot) and contours of $3 \sigma$ statistical significance (lower plot) for the $h \rightarrow \tau^{+} \tau^{-}$channel in CED production in the $M_{A}-\tan \beta$ plane of the MSSM within the "CDM benchmark" scenario P3. The results are shown for assumed effective luminosities (see text, combining ATLAS and CMS) of $60 \mathrm{fb}^{-1}, 60 \mathrm{fb}^{-1}$ eff $\times 2,600$ $\mathrm{fb}^{-1}$ and $600 \mathrm{fb}^{-1} \mathrm{eff} \times 2$. The values of $M_{h}$ are indicated by contour lines. The dark shaded (blue) region corresponds to the parameter region that is excluded by the LEP Higgs searches, the lighter shaded (pink) areas are excluded by Tevatron Higgs searches. 

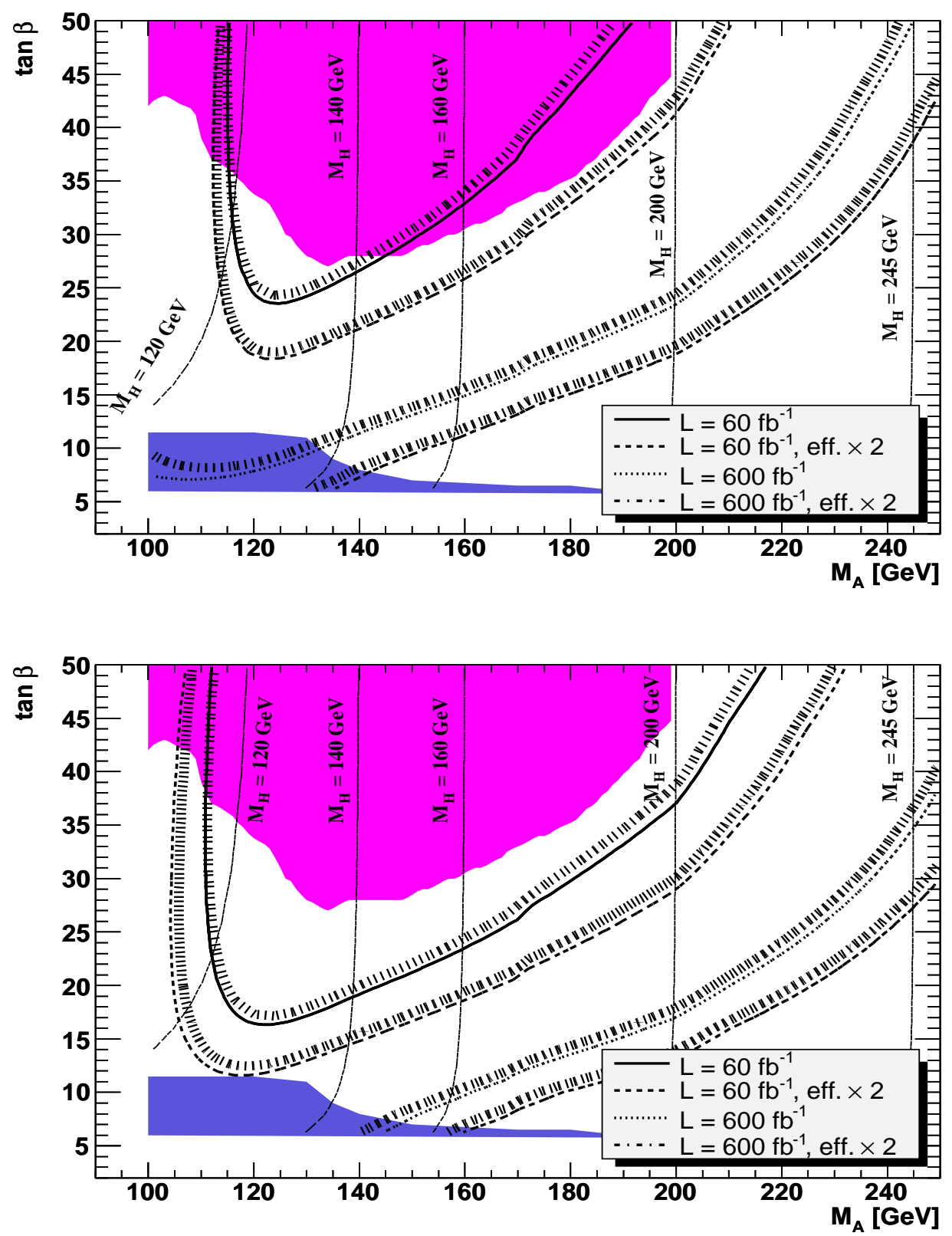

Figure 12: $5 \sigma$ discovery contours (upper plot) and contours of $3 \sigma$ statistical significance (lower plot) for the $H \rightarrow b \bar{b}$ channel in CED production in the $M_{A}-\tan \beta$ plane of the MSSM within the "CDM benchmark" scenario P3. The results are shown for assumed effective luminosities (see text, combining ATLAS and CMS) of $60 \mathrm{fb}^{-1}, 60 \mathrm{fb}^{-1}$ eff $\times 2,600 \mathrm{fb}^{-1}$ and $600 \mathrm{fb}^{-1} \mathrm{eff} \times 2$. The values of $M_{H}$ are indicated by contour lines. The dark shaded (blue) region corresponds to the parameter region that is excluded by the LEP Higgs searches, the lighter shaded (pink) areas are excluded by Tevatron Higgs searches. 

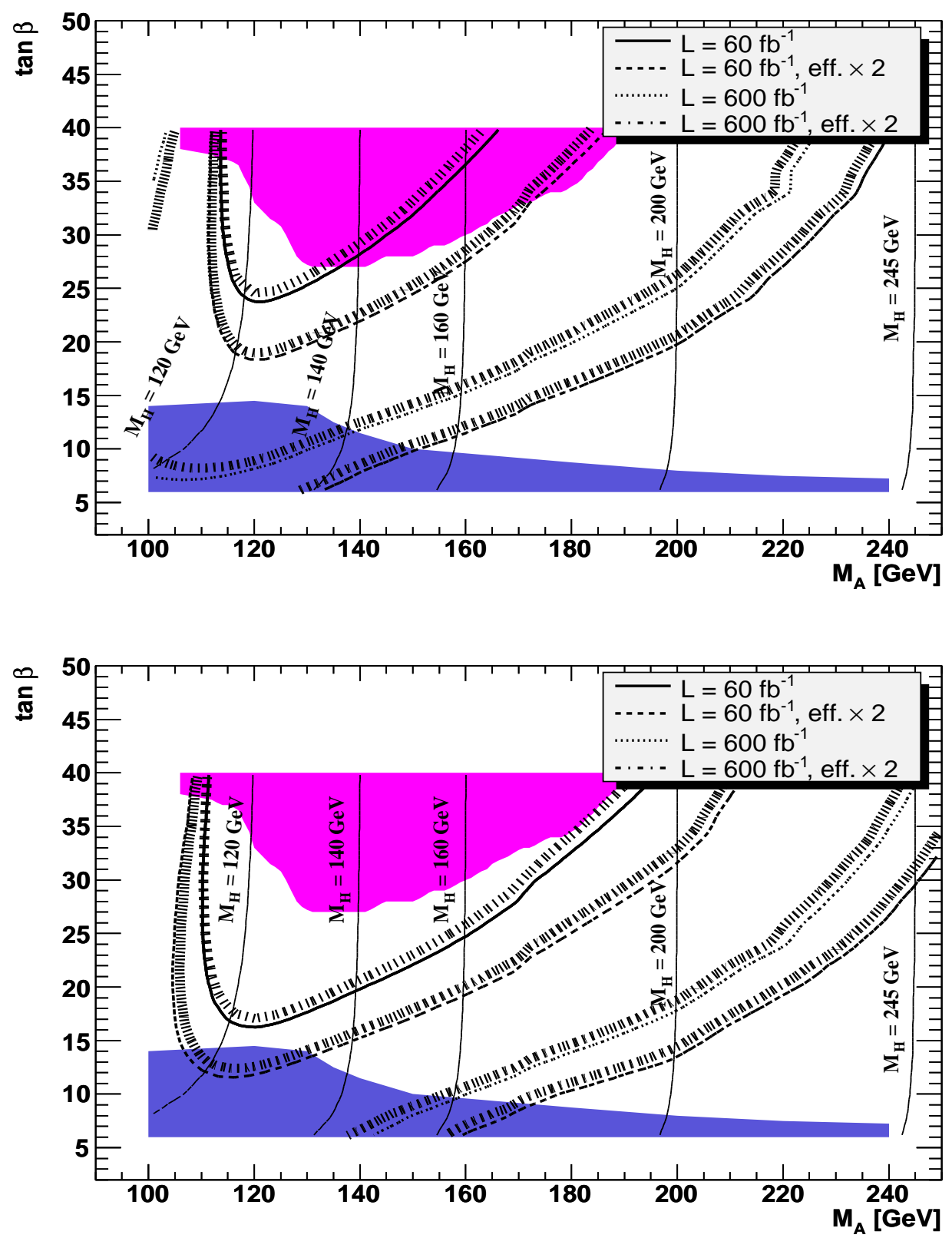

Figure 13: $5 \sigma$ discovery contours (upper plot) and contours of $3 \sigma$ statistical significance (lower plot) for the $H \rightarrow b \bar{b}$ channel in CED production in the $M_{A}-\tan \beta$ plane of the MSSM within the "CDM benchmark" scenario P4. The results are shown for assumed effective luminosities (see text, combining ATLAS and CMS) of $60 \mathrm{fb}^{-1}, 60 \mathrm{fb}^{-1}$ eff $\times 2,600 \mathrm{fb}^{-1}$ and $600 \mathrm{fb}^{-1} \mathrm{eff} \times 2$. The values of $M_{H}$ are indicated by contour lines. The dark shaded (blue) region corresponds to the parameter region that is excluded by the LEP Higgs searches, the lighter shaded (pink) areas are excluded by Tevatron Higgs searches. 

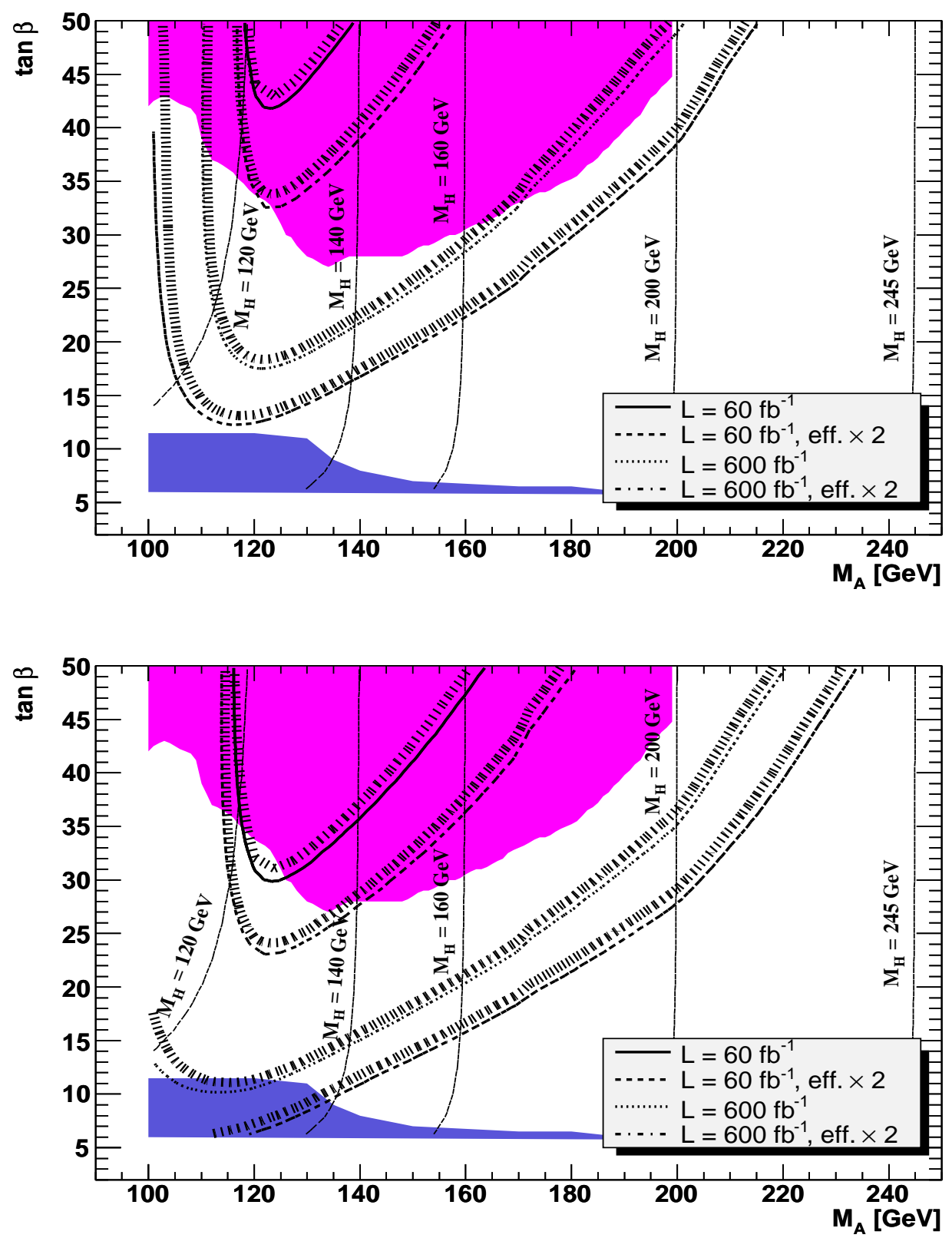

Figure 14: $5 \sigma$ discovery contours (upper plot) and contours of $3 \sigma$ statistical significance (lower plot) for the $H \rightarrow \tau^{+} \tau^{-}$channel in CED production in the $M_{A}$-tan $\beta$ plane of the MSSM within the "CDM benchmark" scenario P3. The results are shown for assumed effective luminosities (see text, combining ATLAS and CMS) of $60 \mathrm{fb}^{-1}, 60 \mathrm{fb}^{-1}$ eff $\times 2,600$ $\mathrm{fb}^{-1}$ and $600 \mathrm{fb}^{-1} \mathrm{eff} \times 2$. The values of $M_{H}$ are indicated by contour lines. The dark shaded (blue) region corresponds to the parameter region that is excluded by the LEP Higgs searches, the lighter shaded (pink) areas are excluded by Tevatron Higgs searches. 
instantaneous luminosity, as assumed in the $600 \mathrm{fb}^{-1}$ and $600 \mathrm{fb}^{-1}$ eff $\times 2$ scenarios, nearly the whole parameter space in the $M_{A}-\tan \beta$ plane can be covered at the $3 \sigma$ level with the searches for CED production of the light and heavy neutral $\mathcal{C} \mathcal{P}$-even Higgs bosons of the MSSM. As discussed in more detail below, the detection of a Higgs candidate in this channel would provide important information about the properties of the observed state.

\section{CED Higgs production in a model with a fourth gen- eration of fermions}

A rather simple example of physics beyond the SM is a model "SM4" which extends the SM by a fourth generation of heavy fermions, see for instance Refs. [22 24]. The masses of the 4th generation quarks in such a scenario need to be significantly heavier than the mass of the top quark 6 As a consequence, the effective coupling of the Higgs boson to two gluons in the SM4 is to good approximation three times larger than in the SM. The effect of the 4th generation on the other couplings that are relevant for Higgs searches at LEP and the Tevatron is small. The phenomenological impact on the Higgs searches can therefore be described by a change of the partial decay width $\Gamma(H \rightarrow g g)$ by a factor of 9 , giving rise to a corresponding shift in the total Higgs width and therefore all the decay branching ratios, see for instance Ref. [25,80]. The total decay width in the SM4 and the relevant decay branching ratios can to good approximation be evaluated in terms of the corresponding quantities in the SM as

$$
\begin{aligned}
\Gamma_{\mathrm{SM}}(H \rightarrow g g) & =\mathrm{BR}_{\mathrm{SM}}(H \rightarrow g g) \Gamma_{\mathrm{tot}}^{\mathrm{SM}}(H), \\
\Gamma_{\mathrm{SM} 4}(H \rightarrow g g) & =9 \Gamma_{\mathrm{SM}}(H \rightarrow g g), \\
\Gamma_{\mathrm{tot}}^{\mathrm{SM} 4}(H) & =\Gamma_{\mathrm{tot}}^{\mathrm{SM}}(H)-\Gamma_{\mathrm{SM}}(H \rightarrow g g)+\Gamma_{\mathrm{SM} 4}(H \rightarrow g g) .
\end{aligned}
$$

Because of the enhanced effective coupling of the Higgs boson to two gluons in the SM4, the Tevatron Higgs searches based in particular on the channel $g g \rightarrow H \rightarrow W W^{(*)}$ have a significantly higher sensitivity as compared to the SM case. In a recent combined analysis of the $\mathrm{CDF}$ and $\mathrm{D} \varnothing$ collaborations the mass range $130 \mathrm{GeV} \lesssim M_{H^{\mathrm{Sm}} 4} \lesssim 210 \mathrm{GeV}$ could be excluded at the $95 \%$ C.L. [81]. The LEP Higgs searches, on the other hand, exclude the Higgs boson of the SM4 for Higgs masses below $\sim 112 \mathrm{GeV}$, see the discussion in Ref. [41. Thus, there remains an unexcluded mass window for a relatively light Higgs in the SM4 of $112 \mathrm{GeV} \lesssim M_{H^{\mathrm{SM} 4}} \lesssim 130 \mathrm{GeV}$. We now investigate the sensitivity of CED Higgs production at the LHC to the Higgs boson of the fourth generation model within this mass window.

As for our analysis within the MSSM discussed above, we have evaluated the significances that can be obtained in the channels $H \rightarrow b \bar{b}$ and $H \rightarrow \tau^{+} \tau^{-}$. The results are shown in Fig. 15 as a function of $M_{H^{\mathrm{SM}} 4}$ for the four luminosity scenarios discussed above. The regions excluded by LEP appear as blue/light grey for low values of $M_{H^{\mathrm{Sm} 4}}$, and regions excluded by the Tevatron appear as red/dark grey for larger values of $M_{H^{\mathrm{Sm} 4}}$. The $b \bar{b}$ channel (upper plot) shows that even at rather low luminosity the remaining window of $112 \mathrm{GeV} \lesssim M_{H^{\mathrm{Sm}} 4} \lesssim 130 \mathrm{GeV}$ can be covered by CED Higgs production. Due to the smallness of $\mathrm{BR}\left(H^{\mathrm{SM} 4} \rightarrow b \bar{b}\right)$ at

${ }^{6}$ The masses of the 4th generation leptons, which are essentially irrelevant for our discussion below, are less restricted and could be as light as about $100 \mathrm{GeV}$. 

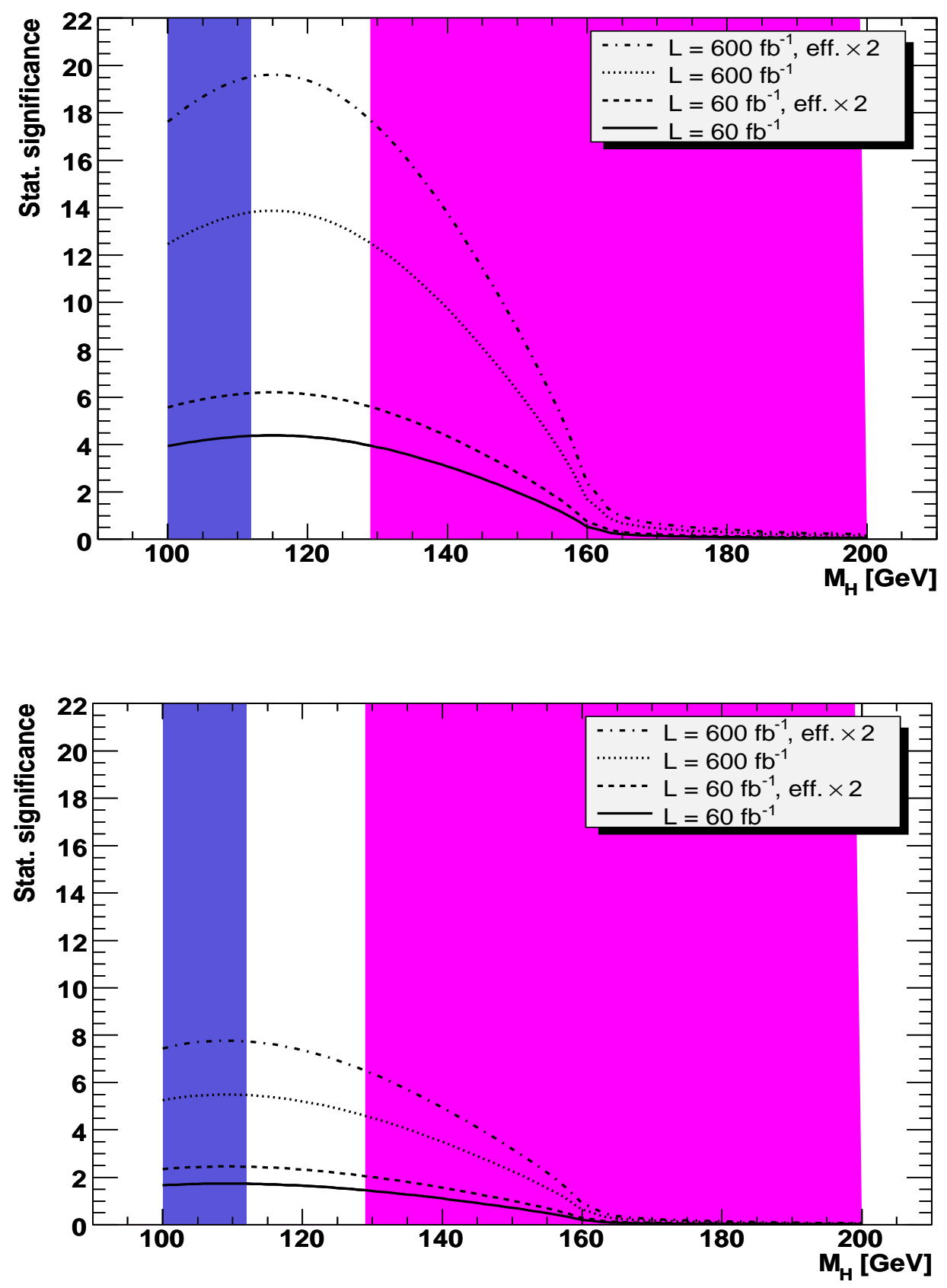

Figure 15: Significances reachable with CED Higgs production in the SM4 in the $H \rightarrow b \bar{b}$ (upper plot) and $H \rightarrow \tau^{+} \tau^{-}$(lower plot) channel for effective luminosities of "60 $\mathrm{fb}^{-1}$ ", "60 fb $\mathrm{fb}^{-1}$ eff $\times 2$ ", "600 $\mathrm{fb}^{-1 "}$ " and "600 $\mathrm{fb}^{-1}$ eff $\times 2$ ". The regions excluded by LEP appear as blue/light grey for low values of $M_{H^{\mathrm{SM}} 4}$ and those excluded by the Tevatron as red/dark grey for larger values of $M_{H^{\mathrm{SM}} 4}$. 
$M_{H^{\mathrm{SM} 4}} \gtrsim 160 \mathrm{GeV}$, however, this channel becomes irrelevant for the still allowed high values of $M_{H^{\mathrm{SM} 4} 4}$, and we do not extend our analysis beyond $M_{H^{\mathrm{SM} 4}} \leq 200 \mathrm{GeV}$. The $\tau^{+} \tau^{-}$ channel (lower plot) reaches a sensitivity of about $2 \sigma$ at low luminosity, while it can exceed the $5 \sigma$ level at high LHC luminosity. At masses $M_{H^{\mathrm{SM}} 4} \gtrsim 220 \mathrm{GeV}$ it might be possible to exploit the decay $H \rightarrow W W, Z Z$, but (apart from the recent analysis in Ref. [82]) no detailed investigation has been performed up to now.

\section{Spin-parity and coupling structure determination of Higgs bosons at the LHC}

The standard methods for determining the spin and the $\mathcal{C P}$ properties of Higgs bosons at the LHC rely to a large extent on the coupling of a relatively heavy Higgs boson to two gauge bosons. In particular, the channel $H \rightarrow Z Z \rightarrow 4 l$ - if it is open - offers good prospects for studying spin and $\mathcal{C P}$-properties of possible Higgs candidates [83] (see also Ref. [84] for a recent analysis of this channel in the context of distinguishing a SM Higgs boson from other possible states of new physics).

Furthermore, the weak boson fusion channel at the LHC has been investigated as a means to obtain information on the coupling of a Higgs-like state to two gauge bosons [85 87]. In a study exploiting the difference in the azimuthal angles of the two tagging jets in weak vector boson fusion [87] it was found that for $M_{H^{\mathrm{SM}}}=160 \mathrm{GeV}$ the decay mode into a pair of $W$-bosons (for which the branching ratio is maximal in this mass range) allows the discrimination between the two extreme scenarios of a pure $\mathcal{C P}$-even (as in the SM) and a pure $\mathcal{C P}$-odd tensor structure at a level of $4.5-5.3 \sigma$ using about $10 \mathrm{fb}^{-1}$ of data (assuming the production rate is that of the SM, which is in conflict with the latest search limits from the Tevatron 63.) A discriminating power of two standard deviations at $M_{H^{\mathrm{SM}}}=120 \mathrm{GeV}$ in the tau lepton decay mode requires an integrated luminosity of $30 \mathrm{fb}^{-1}$ [87.

For $M_{H} \approx M_{A} \gtrsim 2 M_{W}$ the lightest MSSM Higgs boson couples to gauge bosons with about SM strength, but its mass is bounded from above by $M_{h} \lesssim 135 \mathrm{GeV}$ [38], i.e. the light Higgs is in a mass range where the decay to $W W^{(*)}$ or $Z Z^{(*)}$ is difficult to exploit. On the other hand, the heavy MSSM Higgs bosons, $H$ and $A$, decouple from the gauge bosons, see Sect. 2.1. Consequently, since the usually quoted results for the $H \rightarrow Z Z \rightarrow 4 l$ channel and the weak-boson fusion channel with decay into a pair of $W$ bosons assume a relatively heavy $\left(M_{H} \gtrsim 135 \mathrm{GeV}\right)$ SM-like Higgs, these results are not applicable to the case of the MSSM. The same is true for any other model with a light SM-like Higgs and heavy Higgs bosons that decouple from the gauge bosons (it should be noted in this context that in certain parameter regions of general Two-Higgs-Doublet-Models a sizable branching fraction of a heavy scalar or pseudo-scalar Higgs into a pair of gauge bosons is possible Ref. [88]). On the other hand, the analysis mentioned above in the weak boson fusion channel with a subsequent decay into a pair of $\tau$ leptons can be utilised for a relatively light SM-like Higgs and is therefore applicable also to the light $\mathcal{C} \mathcal{P}$-even Higgs boson in the MSSM. However, in the MSSM no significant enhancement of this channel would be expected compared to the SM case, so that the rather modest sensitivities found for the studies in the SM would also hold for the case of the MSSM.

Accordingly, alternative methods for determining the spin and $\mathcal{C P}$-properties of Higgs 
boson candidates that do not rely on the decay into a pair of gauge bosons or on the production in weak boson fusion are of great phenomenological interest. The CED Higgs-boson production process can yield crucial information in this context [2,13, 14, 19]. Because of the $J_{z}=0, C$-even, $P$-even selection rule, which holds for all CED processes, the fact that a certain state is produced in a CED process provides direct information about its spin and $\mathcal{C P}$-properties. It is expected, in particular in a situation where a new particle state has also been detected in one or more of the conventional Higgs search channels, that already a small yield of CED events will be sufficient for extracting relevant information on the spin and $\mathcal{C P}$-properties of the new state.

It should be noted here that most of the existing analyses and also the discussion in the present paper have been performed in the context of the $\mathcal{C} \mathcal{P}$-conserving MSSM, i.e. in terms of the $\mathcal{C P}$ eigenstates $h, H, A$. The CED Higgs-boson analyses can easily be extended to the case where $\mathcal{C} \mathcal{P}$-violating complex phases are present, giving rise to a mixing between the three neutral Higgs bosons. In the general case where the produced state is not necessarily a $\mathcal{C P}$-eigenstate, the selection rule of the CED process can be interpreted as performing a projection of a possibly mixed state onto a $\mathcal{C P}$-eigenstate.

It is worth mentioning that measuring the transverse momentum and azimuthal angle $\phi$ distributions of forward leading protons will provide a unique opportunity to search for a $\mathcal{C P}$-violating signal in the Higgs sector [89]. In particular in some MSSM scenarios with $\mathcal{C P}$-violation the azimuthal asymmetry of the outgoing tagged protons

$$
A=\frac{\sigma(\varphi<\pi)-\sigma(\varphi>\pi)}{\sigma(\varphi<\pi)+\sigma(\varphi>\pi)}
$$

is expected to be quite sizeable. For instance [89,90], $A \simeq 0.07$ can be reached in the scenarios of Refs. [32, 91].

As discussed in Refs. [13, 14] it will be challenging to identify the $\mathcal{C P}$-odd Higgs boson of the MSSM, $A$, in the CED processes because of the strong suppression, caused by the $P$ even selection rule, which effectively filters out its production. However, in the semi-inclusive diffractive reactions the pseudoscalar production is much less suppressed. A recent study in Ref. 5] shows that there are certain advantages of looking for the $\mathcal{C} \mathcal{P}$-odd Higgs particle in the semi-inclusive process $p p \rightarrow p+g A g+p$ with two tagged forward protons and two large rapidity gaps. In this case the amplitude of $\mathcal{C} \mathcal{P}$-odd $A$ boson production is suppressed in comparison with the $\mathcal{C P}$-even boson amplitude only by the momentum fraction $x$ carried by the accompanying gluon. Thus, in the events with one relatively hard gluon, whose energy is comparable with the energy of the whole $g A g$ system, the cross sections of $\mathcal{C} \mathcal{P}$-odd $A$ boson production will be of the same order as that for the $\mathcal{C P}$-even boson.

\section{Conclusions}

We have analysed in this paper the prospects for probing the Higgs sector of models beyond the SM with central exclusive Higgs-boson production processes at the LHC, utilizing forward proton detectors installed at $220 \mathrm{~m}$ and $420 \mathrm{~m}$ distance around ATLAS and CMS. We have updated previous results on the prospects for $\mathrm{CED}$ production of the neutral $\mathcal{C} \mathcal{P}$-even Higgs bosons $h$ and $H$ of the MSSM and their decays into bottom quarks and $\tau$ leptons. With 
respect to Ref. [14 an improved cross section calculation and background evaluation has been performed. Furthermore, the updated exclusion regions from Higgs-boson searches at the Tevatron have been taken into account (and the most complete compilation of the LEP exclusion bounds has been used). In the conventional benchmark scenarios, the $M_{h}^{\max }$ and the no-mixing scenario, we find that the results show a larger coverage in the $M_{A}-\tan \beta$ parameter space in comparison with Ref. [14]. On the other hand, the latest results from the Higgs searches at the Tevatron also yield an enlargement of the excluded regions in the $M_{A}-\tan \beta$, which rule out parts of the parameter regions where the CED Higgs production channels at the LHC have a sensitivity in excess of $5 \sigma$.

We have further extended the results previously obtained for the MSSM by investigating additional "CDM benchmark" scenarios, i.e. $M_{A}-\tan \beta$ planes that are in agreement with the relic abundance of cold dark matter as well as with electroweak precision observables and $B$ physics. As a qualitative result, valid for all analysed benchmark scenarios, we find that at the LHC the CED production channels of the light and heavy $\mathcal{C} \mathcal{P}$-even Higgs bosons of the MSSM with subsequent decay to $b \bar{b}$ and, to a lesser extent, to $\tau^{+} \tau^{-}$provide promising sensitivity. While in the considered scenarios with lower instantaneous luminosity, $60 \mathrm{fb}^{-1}$ and $60 \mathrm{fb}^{-1} \mathrm{eff} \times 2$, some of the $5 \sigma$ regions accessible for the heavy $\mathcal{C} \mathcal{P}$-even Higgs in CED production are excluded by the Tevatron Higgs searches, a significant coverage of currently unexplored parameter space can be achieved at the $3 \sigma$ level. For the $600 \mathrm{fb}^{-1}$ and 600 $\mathrm{fb}^{-1}$ eff $\times 2$ scenarios, which could be realized if the CED channel can be utilized at high instantaneous luminosity, we have demonstrated that the observation of either the light or the heavy $\mathcal{C P}$-even Higgs boson of the MSSM becomes possible at the $3 \sigma$ level in large parts of the MSSM parameter space.

A striking feature of CED Higgs-boson production remains that this channel provides good prospects for detecting Higgs-boson decays into bottom quarks and $\tau$ leptons (and also into $W$ bosons, which has not been updated here). Although the decay into bottom quarks is the dominant decay mode for a light SM-like Higgs boson, this decay channel is difficult to access in the conventional search channels at the LHC. In the MSSM the $b \bar{b}$ and $\tau^{+} \tau^{-}$decay channels are of particular importance, since they are in general dominant even for heavy MSSM Higgs bosons, whereas a SM Higgs boson of the same mass would have a negligible branching ratio into $b \bar{b}$ and $\tau^{+} \tau^{-}$. It should be noted that heavy Higgs bosons that decouple from gauge bosons and therefore predominantly decay into heavy SM fermions (or into other states of new physics, if decays of this kind are kinematically possible) are a quite generic feature of extended Higgs-boson sectors.

As another example of Higgs physics beyond the SM we have analysed the prospects of CED Higgs production in the SM4, i.e. the SM with a fourth generation of (heavy) fermions. In the Higgs mass range below about $200 \mathrm{GeV}$, the LEP and Tevatron Higgsboson searches leave only a relatively small unexcluded window for the SM4 Higgs-boson mass, $112 \mathrm{GeV} \lesssim M_{H^{\mathrm{Sm} 4}} \lesssim 130 \mathrm{GeV}$. We have shown that within this window the CED Higgs production at the LHC with the subsequent decays $H^{\mathrm{SM} 4} \rightarrow b \bar{b}, \tau^{+} \tau^{-}$offers very good prospects for analysing the Higgs sector of the model. For $M_{H^{\mathrm{SM}} 4} \gtrsim 200 \mathrm{GeV}$ the decay to SM gauge bosons becomes dominant, rendering the decay to (light) SM fermions ineffective.

Finally we have discussed the prospects for determining the spin and the coupling structure of possible Higgs candidates at the LHC. The existing analyses assuming a SM-like Higgs boson can only be partially applied to the case of the light $\mathcal{C} \mathcal{P}$-even Higgs boson of 
the MSSM and cannot be employed for the heavy $\mathcal{C P}$-even Higgs boson of the MSSM nor for any other extension of the SM with a light SM-like Higgs and heavy Higgs bosons that decouple from the gauge bosons. We emphasize that the $J_{z}=0, \mathcal{C}$-even, $\mathcal{P}$-even selection rule of the CED Higgs production process leads to a direct information on the properties of any particle produced in this way.

While for clarity of presentation, the results discussed in this paper have been phrased in terms of the $\mathcal{C P}$ eigenstates $h, H, A$ of the Higgs bosons, a generalisation to the general case of arbitrary $\mathcal{C} \mathcal{P}$-violating mixing between the neutral Higgs bosons is easily possible. In this case the selection rule of the CED process has the effect of performing a projection of a possibly mixed state onto a $\mathcal{C P}$ eigenstate. Accordingly, the production of a $\mathcal{C} \mathcal{P}$-odd boson in the CED processes is strongly suppressed. Access to $\mathcal{C} \mathcal{P}$-odd Higgs production could potentially be obtained in semi-inclusive processes. However, further work will be needed to assess the viability of such production modes.

\section{Acknowledgements}

We thank Mike Albrow, Oliver Brein, Peter Bussey, Albert De Roeck, John Ellis, David d'Enterria, Alan Martin, Risto Orava, Krzysztof Piotrzkowski, Christophe Royon, Andy Pilkington, James Stirling and Tim Tait for useful discussions. MGR thanks the IPPP at the University of Durham for hospitality. The work by MGR was supported by the Federal Program of the Russian State RSGSS-3628.2008.2. The work of MT was supported by the project AV0-Z10100502 of the Academy of Sciences of the Czech republic and project LC527 of the Ministry of Education of the Czech republic. The work of SH was partially supported by CICYT (grant FPA 2007-66387). This work is also supported in part by the European Community's Marie-Curie Research Training Network under contract MRTN-CT2006-035505 'Tools and Precision Calculations for Physics Discoveries at Colliders'.

\section{References}

[1] M. Albrow and A. Rostovtsev, arXiv:hep-ph/0009336.

[2] V.A. Khoze, A.D. Martin and M. Ryskin, Eur. Phys. J. C 23 (2002) 311 arXiv:hep-ph/0111078.

[3] for a recent review see M. Albrow, T. Coughlin and J. Forshaw, Prog. Part. Nucl. Phys. 65, 149 (2010) arXiv:1006.1289 [hep-ph]].

[4] A. De Roeck, V.A. Khoze, A. Martin, R. Orava and M. Ryskin, Eur. Phys. J. C 25 (2002) 391 arXiv:hep-ph/0207042.

[5] V.A. Khoze, A. Martin, M. Ryskin and A. Shuvaev, Eur. Phys. J. C 68 (2010) 125 arXiv:1002.2857 [hep-ph]].

[6] CMS and TOTEM diffractive and forward physics working group, CERN/LHCC 2006039/G-124, CMS Note 2007/002, TOTEM Note 06-5, December 2006.

[7] M. Albrow et al., JINST 4 (2009) T10001 [arXiv:0806.0302 [hep-ex]]. 
[8] The AFP project in ATLAS, Letter of Intent, see e.g.:

C. Royon [RP220 Collaboration], arXiv:0706.1796 [physics.ins-det];

M. Tasevsky, Nucl. Phys. Proc. Suppl. 179-180 (2008) 187.

[9] C. Royon, AIP Conf. Proc. 1105 (2009) 130, and references therein.

[10] J. de Favereau de Jeneret et al., arXiv:0908.2020 [hep-ph].

[11] P. Bussey, T. Coughlin, J. Forshaw and A. Pilkington, JHEP 0611 (2006) 027 arXiv:hep-ph/0607264.

[12] M. Tasevsky, arXiv:0910.5205.

[13] A. Kaidalov, V.A. Khoze, A.D. Martin and M. Ryskin, Eur. Phys. J. C 33 (2004) 261 arXiv:hep-ph/0311023.

[14] S. Heinemeyer, V. A. Khoze, M. G. Ryskin, W. J. Stirling, M. Tasevsky and G. Weiglein, Eur. Phys. J. C 53 (2008) 231 [arXiv:0708.3052 [hep-ph]].

[15] B. Cox, F. Loebinger and A. Pilkington, JHEP 0710, 090 (2007) arXiv:0709.3035 [hep$\mathrm{ph}]$.

[16] J. Forshaw et al., JHEP 0804, 090 (2008) arXiv:0712.3510 [hep-ph]].

[17] S. Heinemeyer et al., arXiv:0811.4571 [hep-ph].

[18] M. Chaichian, P. Hoyer, K. Huitu, V. A. Khoze and A. Pilkington, JHEP 0905 (2009) 011 arXiv:0901.3746 [hep-ph]].

[19] S. Heinemeyer, V. A. Khoze, M. Ryskin, M. Tasevsky and G. Weiglein, arXiv:0909.4665 [hep-ph]; arXiv:1009.2680 [hep-ph].

[20] H. Nilles, Phys. Rept. 110 (1984) 1;

H. Haber and G. Kane, Phys. Rept. 117 (1985) 75;

R. Barbieri, Riv. Nuovo Cim. 11 (1988) 1.

[21] M. Carena and H. Haber, Prog. Part. Nucl. Phys. 50 (2003) 63 arXiv:hep-ph/0208209];

S. Heinemeyer, Int. J. Mod. Phys. A 21 (2006) 2659 arXiv:hep-ph/0407244;

S. Heinemeyer, W. Hollik and G. Weiglein, Phys. Rept. 425 (2006) 265 arXiv:hep-ph/0412214;

A. Djouadi, Phys. Rept. 457 (2008) 1 arXiv:hep-ph/0503172]; Phys. Rept. 459 (2008) 1 arXiv:hep-ph/0503173.

[22] P. Frampton, P. Hung and M. Sher, Phys. Rept. 330 (2000) 263 arXiv:hep-ph/9903387.

[23] For a recent review see: B. Holdom, W. Hou, T. Hurth, M. Mangano, S. Sultansoy and G. Unel, PMC Phys. A 3 (2009) 4 [arXiv:0904.4698 [hep-ph]].

[24] J. Erler and P. Langacker, Phys. Rev. Lett. 105 (2010) 031801 arXiv:1003.3211 [hep$\mathrm{ph}]$. 
[25] G. Kribs, T. Plehn, M. Spannowsky and T. Tait, Phys. Rev. D 76 (2007) 075016 arXiv:0706.3718 [hep-ph]].

[26] V. Novikov, A. Rozanov and M. Vysotsky, Phys. Atom. Nucl. 73 (2010) 636 (2010) arXiv:0904.4570 [hep-ph]].

[27] V.A. Khoze, A. Martin and M. Ryskin, Eur. Phys. J. C 19 (2001) 477 [Erratum-ibid. C 20 (2001) 599] arXiv:hep-ph/0011393].

[28] M. Dührssen, S. Heinemeyer, H. Logan, D. Rainwater, G. Weiglein and D. Zeppenfeld, Phys. Rev. D 70 (2004) 113009 arXiv:hep-ph/0406323]; arXiv:hep-ph/0407190].

[29] R. Lafaye, T. Plehn, M. Rauch, D. Zerwas and M. Dührssen, JHEP 0908 (2009) 009 arXiv:0904.3866 [hep-ph]].

[30] V. A. Khoze, A. Martin and M. Ryskin, Eur. Phys. J. C 34 (2004) 327 arXiv:hep-ph/0401078.

[31] J. Ellis, J. Lee and A. Pilaftsis, Nucl. Phys. B 718 (2005) 247 arXiv:hep-ph/0411379.

[32] J. Ellis, J. Lee and A. Pilaftsis, Phys. Rev. D 71 (2005) 075007 arXiv:hep-ph/0502251].

[33] M. Boonekamp, J. Cammin, S. Lavignac, R. Peschanski and C. Royon, Phys. Rev. D 73 (2006) 115011 arXiv:hep-ph/0506275].

[34] A. Shuvaev et al., Eur. Phys. J. C 56, 467 (2008) [arXiv:0806.1447 [hep-ph]].

[35] V. A. Khoze, A. Martin and M. Ryskin, Eur. Phys. J. C 64 (2009) 361 arXiv:0907.0966 [hep-ph]].

[36] S. Heinemeyer, W. Hollik and G. Weiglein, Comp. Phys. Commun. 124200076 arXiv:hep-ph/9812320].

[37] S. Heinemeyer, W. Hollik and G. Weiglein, Eur. Phys. J. C 9 (1999) 343 arXiv:hep-ph/9812472.

[38] G. Degrassi, S. Heinemeyer, W. Hollik, P. Slavich and G. Weiglein, Eur. Phys. J. C 28 (2003) 133 arXiv:hep-ph/0212020].

[39] M. Frank, T. Hahn, S. Heinemeyer, W. Hollik, H. Rzehak and G. Weiglein, JHEP 0702 (2007) 047 arXiv:hep-ph/0611326.

[40] See: www.feynhiggs.de .

[41] P. Bechtle, O. Brein, S. Heinemeyer, G. Weiglein and K. Williams, Comput. Phys. Commun. 181 (2010) 138 [arXiv:0811.4169 [hep-ph]]; arXiv:0909.4664 [hep-ph]; the code can be obtained via www.ippp.dur.ac.uk/HiggsBounds .

[42] M. Carena, S. Heinemeyer, C. Wagner and G. Weiglein, Eur. Phys. J. C 26 (2003) 601 arXiv:hep-ph/0202167]. 
[43] R. Hemplling, Phys. Rev. D 49 (1994) 6168;

L. Hall, R. Rattazzi and U. Sarid, Phys. Rev. D 50 (1994) 7048 arXiv:hep-ph/9306309;; M. Carena, M. Olechowski, S. Pokorski and C. Wagner, Nucl. Phys. B 426 (1994) 269 arXiv:hep-ph/9402253.

[44] M. Carena, D. Garcia, U. Nierste and C. Wagner, Nucl. Phys. B 577 (2000) 577 arXiv:hep-ph/9912516.

[45] H. Eberl, K. Hidaka, S. Kraml, W. Majerotto and Y. Yamada, Phys. Rev. D 62 (2000) 055006 arXiv:hep-ph/9912463].

[46] J. Guasch, P. Häfliger and M. Spira, Phys. Rev. D 68 (2003) 115001 arXiv:hep-ph/0305101.

[47] A. Brignole, G. Degrassi, P. Slavich and F. Zwirner, Nucl. Phys. B 643 (2002) 79 arXiv:hep-ph/0206101.

[48] G. Degrassi, A. Dedes and P. Slavich, Nucl. Phys. B 672 (2003) 144 arXiv:hep-ph/0305127].

[49] S. Heinemeyer, W. Hollik, H. Rzehak and G. Weiglein, Eur. Phys. J. C 39 (2005) 465 arXiv:hep-ph/0411114]; arXiv:hep-ph/0506254.

[50] D. Noth and M. Spira, Phys. Rev. Lett. 101 (2008) 181801 [arXiv:0808.0087 [hep-ph]].

[51] L. Hofer, U. Nierste and D. Scherer, JHEP 0910 (2009) 081 [arXiv:0907.5408 [hep-ph]].

[52] A. Martin, M. Ryskin and G. Watt, Eur. Phys. J. C 66 (2010) 163 arXiv:0909.5529 [hep-ph]].

[53] T. Coughlin and J. Forshaw, JHEP 1001 (2010) 121 [arXiv:0912.3280 [hep-ph]].

[54] A. Martin, M. Ryskin and V. A. Khoze, Acta Phys. Polon. B 40 (2009) 1841 arXiv:0903.2980 [hep-ph]].

[55] M. Ryskin, A. Martin and V. A. Khoze, Eur. Phys. J. C 60 (2009) 265 arXiv:0812.2413 [hep-ph]].

[56] M. Ryskin, A. Martin and V. A. Khoze, arXiv:1102.2844 [hep-ph].

[57] V. A. Khoze, A. Martin and M. Ryskin, Eur. Phys. J. C 55 (2008) 363 arXiv:0802.0177 [hep-ph]].

[58] L. Bonnet, T. Pierzchala, K. Piotrzkowski and P. Rodeghiero, Acta Phys. Polon. B 38 (2007) 477 arXiv:hep-ph/0703320].

[59] K. Piotrzkowski and N. Schul, AIP Conf. Proc. 1200 (2010) 434 arXiv:0910.0202 [hep$\mathrm{ph}]$.

[60] M. Carena, S. Heinemeyer, C. Wagner and G. Weiglein, Eur. Phys. J. C 45 (2006) 797 arXiv:hep-ph/0511023. 
[61] G. Abbiendi et al. [ALEPH, DELPHI, L3, OPAL Collaborations and LEP Working Group for Higgs boson searches], Phys. Lett. B 565 (2003) 61 [arXiv:hep-ex/0306033.

[62] S. Schael et al. [ALEPH, DELPHI, L3, OPAL Collaborations and LEP Working Group for Higgs boson searches], Eur. Phys. J. C 47 (2006) 547 arXiv:hep-ex/0602042.

[63] See for the latest results:

[CDF Collaboration and DØ Collaboration], arXiv:1007.4587 [hep-ex];

T. Aaltonen et al. [CDF and D $\varnothing$ Collaborations], Phys. Rev. Lett. 104 (2010) 061802 arXiv:1001.4162 [hep-ex]].

[64] See for the latest results:

D. Benjamin et al. [Tevatron New Phenomena and Higgs Working Group], arXiv:1003.3363 [hep-ex];

[CDF Collaboration], CDF Note 10105;

[D $\varnothing$ collaboration], DØ Note 6083-Conf;

T. Aaltonen et al. [CDF Collaboration], Phys. Rev. Lett. 103 (2009) 101803 arXiv:0907.1269 [hep-ex]].

[65] S. Heinemeyer, W. Hollik and G. Weiglein, JHEP 0006 (2000) 009 arXiv:hep-ph/9909540].

[66] J. Ellis, S. Heinemeyer, K. Olive, A. Weber, G. Weiglein, JHEP 0708 (2007) 083 arXiv:0706.0652 [hep-ph]].

[67] J. Ellis, T. Hahn, S. Heinemeyer, K. Olive and G. Weiglein, JHEP 0710 (2007) 092 arXiv:0709.0098 [hep-ph]].

[68] M. Olechowski and S. Pokorski, Phys. Lett. B 344 (1995) 201 arXiv:hep-ph/9407404; V. Berezinsky, A. Bottino, J. Ellis, N. Fornengo, G. Mignola and S. Scopel, Astropart. Phys. 5 (1996) 1 arXiv:hep-ph/9508249|;

M. Drees, M. Nojiri, D. Roy and Y. Yamada, Phys. Rev. D 56 (1997) 276 [Erratum-ibid. D 64 (1997) 039901] arXiv:hep-ph/9701219];

M. Drees, Y. Kim, M. Nojiri, D. Toya, K. Hasuko and T. Kobayashi, Phys. Rev. D 63 (2001) 035008 arXiv:hep-ph/0007202;

P. Nath and R. Arnowitt, Phys. Rev. D 56 (1997) 2820 arXiv:hep-ph/9701301;

J. Ellis, T. Falk, K. Olive and Y. Santoso, Nucl. Phys. B 652 (2003) 259 arXiv:hep-ph/0210205];

H. Baer, A. Mustafayev, S. Profumo, A. Belyaev and X. Tata, JHEP 0507 (2005) 065 arXiv:hep-ph/0504001].

[69] C. Bennett et al., Astrophys. J. Suppl. 148 (2003) 1 [arXiv:astro-ph/0302207];

D. Spergel et al. [WMAP Collaboration], Astrophys. J. Suppl. 148 (2003) 175 arXiv:astro-ph/0302209];

D. Spergel et al. [WMAP Collaboration], Astrophys. J. Suppl. 170 (2007) 377 arXiv:astro-ph/0603449.

[70] R Harlander and W. Kilgore, Phys. Rev. D 68 (2003) 013001 arXiv:hep-ph/0304035. 
[71] A. Martin, R. Roberts, W. Stirling and R. Thorne, Eur. Phys. J. C 28 (2003) 455 arXiv:hep-ph/0211080.

[72] A. Martin, W. Stirling, R. Thorne and G. Watt, Eur. Phys. J. C 63 (2009) 189 arXiv:0901.0002 [hep-ph]].

[73] G. Aad et al. [The ATLAS Collaboration], "Expected Performance of the ATLAS Experiment - Detector, Trigger and Physics," arXiv:0901.0512.

[74] G. Bayatian et al. [CMS Collaboration], CMS Technical Design Report, Volume II: Physics Performance, CERN-LHCC-2006-021, CMS-TDR-008-2, J. Phys. G 34 (2007) 995 (2007); see: http://cmsdoc.cern.ch/cms/cpt/tdr/.

[75] J. Butterworth, A. Davison, M. Rubin and G. Salam, Phys. Rev. Lett. 100 (2008) 242001 [arXiv:0802.2470 [hep-ph]].

[76] A. De Roeck et al., Eur. Phys. J. C 66 (2010) 525 [arXiv:0909.3240 [hep-ph]].

[77] M. Schumacher, Czech. J. Phys. 54 (2004) A103; hep-ph/0410112.

[78] S. Abdullin et al., Eur. Phys. J. C 39S2 (2005) 41.

[79] S. Gennai, S. Heinemeyer, A. Kalinowski, R. Kinnunen, S. Lehti, A. Nikitenko and G. Weiglein, Eur. Phys. J. C 52 (2007) 383 [arXiv:0704.0619 [hep-ph]].

[80] C. Anastasiou, R. Boughezal and E. Furlan, JHEP 1006 (2010) 101 arXiv:1003.4677 [hep-ph]].

[81] TEVNPH Working Group Collaboration for the CDF Collaboration and D $\varnothing$ Collaboration, CDF note 10101, DØ note 6039.

[82] C. Hackstein and M. Spannowsky, arXiv:1008.2202 [hep-ph].

[83] V. Buescher and K. Jakobs, Int. J. Mod. Phys. A 20 (2005) 2523 arXiv:hep-ph/0504099].

[84] A. De Rujula, J. Lykken, M. Pierini, C. Rogan and M. Spiropulu, Phys. Rev. D 82 (2010) 013003 arXiv:1001.5300 [hep-ph]].

[85] T. Plehn, D. L. Rainwater and D. Zeppenfeld, Phys. Rev. Lett. 88 (2002) 051801 arXiv:hep-ph/0105325.

[86] V. Hankele, G. Klamke, D. Zeppenfeld and T. Figy, Phys. Rev. D 74 (2006) 095001 arXiv:hep-ph/0609075].

[87] C. Ruwiedel, N. Wermes and M. Schumacher, Eur. Phys. J. C 51 (2007) 385.

[88] W. Bernreuther, P. Gonzalez and M. Wiebusch, arXiv:1003.5585 [hep-ph].

[89] V.A. Khoze, A. Martin and M.G. Ryskin, Eur. Phys. J. 34 (2004) 327 arXiv:hep-ph/0401078]. 
[90] V.A. Khoze, A. Martin and M.G. Ryskin in: E. Accomando et al., arXiv:hep-ph/0608079.

[91] M. Carena, J. Ellis, A. Pilaftsis and C. Wagner, Phys. Lett. B 495 (2000) 155, hep-ph/0009212. 